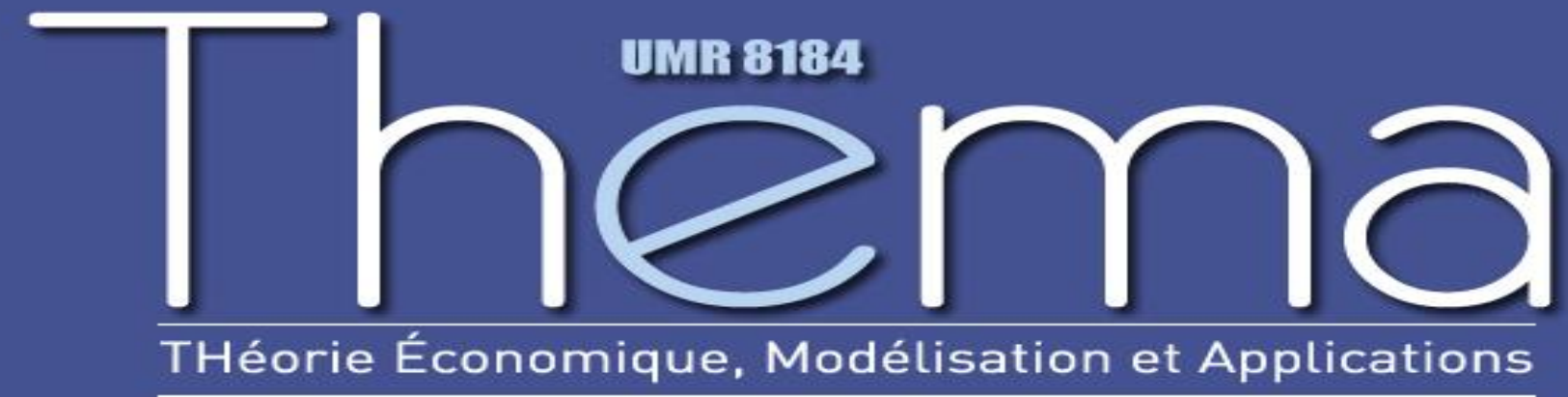

Thema Working Paper $\mathrm{n}^{\circ}$ 2014-04 Université de Cergy Pontoise, France

"Les inégalités intra-familiales d'éducation en France"

Nathalie Picard

François-Charles Wolff

March, 2014 


\title{
Les inégalités intra-familiales d'éducation en France
}

\author{
Nathalie Picard* \\ François-Charles Wolff**
}

\begin{abstract}
Si la transmission intergénérationnelle du capital humain a été largement étudiée par les économistes, on sait, en revanche, peu de choses sur les écarts scolaires entre frères et sœurs. Cet article propose une mesure de l'hétérogénéité d'éducation entre les enfants pour la France sur la base d'un échantillon comprenant 27197 enfants appartenant à 11694 familles. L'estimation de modèles Probit ordonnés à effets aléatoires et la mise en œuvre d'une décomposition de variance permettent de distinguer l'hétérogénéité d'éducation entre les familles de celle au sein des familles, entre frères et sœurs. Les résultats indiquent que l'hétérogénéité d'éducation entre les familles représente près de deux tiers de la variabilité totale. Au sein des fratries, les filles et les aînés sont relativement plus éduqués.
\end{abstract}

\section{EDUCATIONAL INEQUALITIES WITHIN FAMILIES IN FRANCE}

While intergenerational transmission of human capital has been widely documented by economists, less is known about differences in schooling between siblings. This paper proposes a measure of educational inequality in France using a sample of 27,197 children from 11,694 families. Estimation of ordered Probit models with random effects and application of variance decomposition allow making a distinction between educational inequalities between families and educational inequalities within families, among siblings. Results show that differences in schooling between families amount to about two-thirds of the total variance. Compared to their siblings, girls and first-born children are more educated.

Classification JEL: D13, I21

* THEMA, Université de Cergy-Pontoise et École polytechnique. Correspondance : THEMA, 33 boulevard du Port, 95011 Cergy-Pontoise Cedex. Courriel : nathalie.picard@u-cergy.fr

** LEMNA, Université de Nantes et INED, Paris. Correspondance : LEMNA, BP 52231, chemin de la Censive du Tertre, 44322 Nantes Cedex 3, France. Courriel : francois.wolff@univ-nantes.fr (auteur correspondant). Site Web : www.sc-eco.univ-nantes.fr/ fcwolff

Nous tenons à remercier deux rapporteurs anonymes pour leurs nombreuses remarques et suggestions sur des versions précédentes de ce texte ainsi que les participants à la $3^{\text {e }}$ Rencontre scientifique annuelle de l'Instance nationale d'évaluation du Conseil supérieur de l'enseignement du Maroc (Rabat, avril 2010) et au $2^{\text {nd }}$ Workshop du Centre de recherche sur le capital humain (Lille, juin 2012). Cette recherche a bénéficié du soutien financier de la Caisse nationale des allocations familiales. 


\section{INTRODUCTION}

De très nombreuses études économétriques se sont intéressées aux déterminants du niveau éducatif des enfants (Björklund et Salvanes [2011]). Cellesci mettent en évidence deux grands types de variables familiales qui viennent influencer la réussite scolaire.

D'une part, le diplôme dépend du milieu parental dans lequel évoluent les enfants. Avoir des parents diplômés ou appartenir à des familles fortunées sont des éléments qui favorisent sensiblement l'acquisition de capital humain (Black et al. [2005a]). D'autre part, la réussite scolaire est fonction des propres caractéristiques des enfants. Les garçons et les filles n'ont pas toujours les mêmes chances de réussite à l'école, et le diplôme dépend aussi de la composition de la fratrie dont sont issus les enfants. Avoir de nombreux frères et sœurs, avoir des frères plutôt que des sœurs à taille de fratrie donnée, ou faire partie des derniersnés constituent, dans certains pays, des freins à la réussite scolaire (Black et al. [2005b] ; Booth et Kee [2009] ; Butcher et Case [1994]) ${ }^{1}$.

Les caractéristiques de l'enfant et de sa famille ne sont pas les seules à influencer sa trajectoire scolaire. Les investissements publics et, plus généralement, le contexte institutionnel viennent également modifier le niveau éducatif, que ce soit par l'accueil des plus jeunes enfants dans des écoles maternelles, par la modulation de la taille des classes ou bien encore par la mise en concurrence des établissements supérieurs (Hanushek et Woessmann [2011]). La combinaison de tous ces éléments donne lieu à de multiples inégalités dans la réussite scolaire, que ce soit entre les pays, au sein des différents pays avec des disparités régionales ou des différences entre les milieux urbains et ruraux, entre les familles, ou au sein même des familles entre les frères et sœurs.

Curieusement, les études empiriques qui se sont intéressées aux inégalités intra-familiales d'éducation demeurent peu nombreuses (Behrman et al. [1995] ; Erjnaes et Pörtner [2004] ; Picard et Wolff [2010])². La littérature économique nous enseigne pourtant qu'il est très vraisemblable que l'hétérogénéité d'éducation au sein des fratries ne soit pas négligeable du tout. Pour diverses raisons, qui ne résultent pas toujours de choix délibérés des parents, certains enfants dans les fratries peuvent mieux réussir que leurs frères et sœurs.

Les parents peuvent tout d'abord délibérément favoriser le capital humain de certains de leurs enfants. S'ils ont par exemple une préférence pour les garçons ou bien pour les aînés, il est vraisemblable qu'ils consacreront davantage de ressources (sous forme d'argent ou de temps) pour leur éducation. Il se peut aussi qu'ils soient amenés à faire des choix parce qu'ils n'ont pas suffisamment de ressources financières pour aider dans des proportions similaires l'ensemble de leurs enfants. S'ils sont contraints par la liquidité, les parents peuvent alors

1. Il existe de nombreuses controverses autour des effets de la composition de la fratrie. Ainsi, pour les États-Unis, Kaestner [1997] et Hauser et Kuo [1998] ne trouvent pas d'effet significatif de la composition par sexe, contrairement à Butcher et Case [1994]. En ce qui concerne le rang de naissance, Black et al. [2005b] montrent que sa prise en compte dans les régressions fait disparaître l'effet négatif du nombre de frères et sœurs sur le diplôme en Norvège, mais Booth et Kee [2009] ne trouvent pas une telle relation en Angleterre.

2. Behrman [1997] propose une revue détaillée des modèles d'allocation des ressources au sein de la famille. 
investir dans les enfants caractérisés par les rendements éducatifs les plus élevés (Garg et Morduch [1998]).

Dans certains cas, les mécanismes à l'origine d'inégalités entre collatéraux ne sont pas délibérés. Les derniers-nés dans les fratries peuvent ainsi être favorisés puisque leurs parents vont avoir plus de ressources à leur consacrer une fois les aînés éduqués. Il en sera de même si les revenus des parents augmentent au cours de leur cycle de vie à un rythme plus élevé que ne s'accroissent les frais de scolarité. Dans le même temps, le fait d'être parmi les premiers-nés peut s'avérer bénéfique puisque les aînés vivent plus longtemps que les cadets au sein d'une fratrie de petite taille, si bien que l'effet du rang est indéterminé dans le cas général (Wolff [2012]). Enfin, il peut y avoir des changements dans la sélectivité du système éducatif au cours du temps, qui peuvent favoriser les générations les plus récentes (Magnac et Thesmar [2002]).

Dans cet article, nous nous intéressons aux inégalités d'éducation au sein des fratries en France, c'est-à-dire à l'hétérogénéité d'éducation entre frères et sœurs. La démarche est ici positive. Il s'agit d'apprécier l'importance des écarts de diplôme entre les enfants d'une même famille et non de savoir si le décideur public doit poursuivre un objectif de méritocratie ou bien de démocratisation du système scolaire ${ }^{3}$.

Nous cherchons donc à mesurer l'hétérogénéité d'éducation en distinguant les inégalités qui existent entre les familles de celles présentes au sein des familles entre les différents frères et sœurs. Nous mobilisons à cet effet trois enquêtes s'intéressant au patrimoine des ménages qui ont été réalisées par l'Insee en 1992, 1998 et 2004. Ces données permettent d'étudier l'allocation intra-familiale de l'éducation puisque nous connaissons le niveau d'éducation de chaque enfant et que nous avons accès à une description complète de la fratrie (nombre d'enfants, composition par sexe, rang de naissance de chaque enfant $)^{4}$. Les enfants étudiés sont, pour la plupart, nés entre 1945 et 1980.

Notre analyse économétrique repose sur l'estimation de modèles ordonnés à effets aléatoires pour rendre compte des niveaux éducatifs des frères et sœurs. Suivant la méthodologie proposée par Picard et Wolff [2010], nous procédons à des décompositions de variance sur le niveau d'éducation latent de chaque enfant, obtenu à partir d'un modèle ordonné. Les résultats que nous obtenons indiquent que les inégalités d'éducation entre les familles, qu'elles soient expliquées ou inexpliquées, représentent près de deux tiers de la variance totale. Il existe donc une hétérogénéité substantielle dans les diplômes entre les frères et sœurs. Au sein des fratries, ce sont les filles et les aînés qui s'en sortent relativement le mieux.

3. Nous remercions un rapporteur anonyme pour ses commentaires sur ce point. Si une forte hétérogénéité d'éducation entre frères et sœurs est compatible avec un système scolaire méritocratique, un système scolaire, fondé sur des inégalités de naissance, conduit à une homogénéité totale des niveaux d'éducation au sein des familles. Un tel débat ne saurait être tranché avec les données que nous mobilisons, notamment parce que nous ne sommes pas en mesure de distinguer les caractéristiques héritées par les enfants de leurs parents de leur effort produit pour réussir leur parcours scolaire.

4. Si ces enquêtes permettent de mesurer l'hétérogénéité d'éducation entre frères et sœurs, il importe de préciser que ces enquêtes n’ont pas été conçues pour étudier les trajectoires scolaires des enfants. Ainsi, l'information recueillie sur les diplômes ne permet pas de connaître avec précision les filières suivies ou bien les éventuels redoublements intervenus. 
La suite de cet article est organisée de la façon suivante. La section suivante propose une synthèse des travaux sur le lien entre la réussite scolaire et les caractéristiques familiales en France. Ensuite, nous décrivons les bases de données utilisées et précisons l'évolution du niveau de diplôme des enfants par cohorte de naissance. La spécification économétrique retenue est présentée dans une quatrième section. Les déterminants du niveau de diplôme des enfants et le poids des inégalités d'éducation entre les familles et au sein des fratries sont discutés dans la cinquième section. Enfin, une dernière section conclut.

\section{REVUE DE LITTÉRATURE}

Analysant le lien entre l'origine sociale et la destinée scolaire sur la base de quatre enquêtes sur la formation et la qualification professionnelle (de 1970 à 1993), Goux et Maurin [1995] montrent que le développement du système éducatif dans la période d'après guerre s'est accompagné d'une ouverture à des milieux sociaux qui en étaient largement exclus jusqu'alors. Toutefois, si la démocratisation de l'enseignement a profité aux enfants des familles les moins qualifiées, elle a aussi bénéficié aux enfants issus des classes intermédiaires. Dès lors, cette démocratisation n'est pas venue réduire les inégalités observées, puisque le schéma observé est proche d'une démocratisation uniforme où tous les enfants sont concernés ${ }^{5}$.

Goux et Maurin [1997] mettent en avant l'importance croissante des facteurs culturels par rapport aux facteurs socioéconomiques pour rendre compte de la persistance des inégalités scolaires entre les enfants d'une même génération. Une meilleure connaissance du système scolaire, toujours plus complexe, par les parents les plus diplômés vient naturellement favoriser les parcours de leurs enfants. Le lien entre les hiérarchies scolaires des enfants et des parents tend ainsi à se renforcer au fil des générations (Goux et Maurin [1995]). Plusieurs études ont cherché à évaluer cette intensité de la transmission intergénérationnelle du capital humain, en mettant simplement en relation la dotation en capital humain d'un individu avec celle de ses parents ${ }^{6}$.

Utilisant les données de l'enquête Formation et qualification professionnelle 2003, Fabre et Moullet [2004] proposent des estimations de la corrélation entre le capital humain des parents et celui des enfants (mesuré par la durée de scolarité), tout en tenant compte de la dotation en capital humain moyen de la société. Elles obtiennent des coefficients de transmission intergénérationnelle du capital humain de l'ordre de 0,3 à la fois pour les durées d'études du père et de la mère. Ce résultat ne permet cependant pas de déduire que le fait d'avoir des parents diplômés a un effet causal sur la réussite scolaire des enfants (Card [2001]). Le lien statistique mis en évidence entre les niveaux de capital humain des deux

5. Albouy et Tavan [2008] s'intéressent plus spécifiquement à la démocratisation de l'enseignement supérieur sur la base des enquêtes Emploi de 1990 à 2002. Si la démocratisation de l'enseignement supérieur a été de même ampleur pour ses différents niveaux de diplôme, elle apparaît moins prononcée que celle du baccalauréat.

6. Pour une revue des travaux empiriques sur la transmission intergénérationnelle du capital humain au niveau international, se reporter à Becker et Tomes [1986] et à Haveman et Wolfe (1995]. 
générations peut résulter de facteurs inobservables propres aux parents les plus diplômés, qui viendraient jouer sur la trajectoire de leurs enfants.

Pour savoir si cet effet de l'éducation des parents sur celle des enfants est causal ou non, Maurin et McNally [2008] utilisent de manière originale le recours à l'épisode de mai 1968 qui a permis à certaines cohortes d'accéder plus facilement aux études supérieures. Pour des tailles de cohortes de naissance comparable, le nombre de candidats reçus au bac en 1968 a en effet été de $30 \%$ supérieur par rapport aux années adjacentes. Pour celles et ceux qui en ont bénéficié, les auteurs montrent que ce surcroît d'éducation a amélioré non seulement leur propre réussite professionnelle (avec notamment des revenus supérieurs), mais aussi la trajectoire scolaire de leurs enfants. Cet effet causal de l'éducation des parents est tout à fait significatif, puisque chaque année supplémentaire passée par les parents à l'université réduit d'environ $30 \%$ la probabilité pour les enfants de redoubler au primaire ou au collège.

Les enfants issus de milieux défavorisés sur le plan économique s'en sortent également moins bien que les autres. Maurin [2002] étudie l'effet du revenu parental sur la probabilité de redoubler à l'école élémentaire. Il met en évidence un fort effet de la pauvreté des parents sur la réussite scolaire, qui excède largement celui (positif) relevant de l'éducation des parents. Ainsi, une hausse de $10 \%$ du revenu parental donne lieu à une diminution de 6,5 points de la probabilité de redoubler à l'école primaire ${ }^{7}$. Duée [2005] propose une mesure de l'importance du chômage des parents sur le devenir des enfants. Les données de l'enquête Budget des familles 2001 révèlent que les enfants dont le père a connu le chômage ont une probabilité beaucoup plus faible (de l'ordre de 20 points) d'obtenir le bac.

À caractéristiques parentales données, plusieurs variables propres aux enfants influencent leurs trajectoires scolaires. À partir de données longitudinales sur deux cohortes d'élèves, Establet [1988] met en évidence la progression très forte de la réussite scolaire des filles dans tous les milieux sociaux et qualifie ce phénomène de « subversion dans la reproduction scolaire ». Baudelot et Establet [1992] expliquent la meilleure réussite des filles par une socialisation précoce différente de celle des garçons, ce qui peut expliquer les phénomènes d'autoélimination des filles observés pour les filières d'excellence. Si les inégalités sexuées se sont renversées au cours du XX $\mathrm{X}^{\mathrm{e}}$ siècle, les inégalités sociales n'en restent pas moins d'une ampleur beaucoup plus grande sur cette période. La hiérarchie des inégalités sociales apparaît alors assez similaire pour les deux sexes d'après les enquêtes sur la formation et la qualification professionnelle (Duru-Bellat et al. [2001]).

La trajectoire scolaire est également influencée par la fratrie d'appartenance. Par exemple, l'âge de fin de scolarité diminue avec le nombre de frères et sœurs (Barnet-Verzat et Wolff [2003]), et être issu d'une fratrie de grande taille vient réduire fortement le risque d'avoir redoublé avant d'entrer au collège (Maurin [2002]). Le fait que les enfants ayant de nombreux frères et sœurs réussissent moins bien à l'école s'explique principalement par le rôle des conditions de logement : partager sa chambre au moment de l'adolescence vient peser sur les résultats scolaires (Goux et Maurin [2005]). Les effets de la composition par sexe de la fratrie

7. Goux et Maurin [2000] mettent en évidence des résultats similaires sur le retard scolaire des enfants en troisième. 
et du rang sur la réussite apparaissent plus ambigus. Si Duée [2005] note que ces deux variables n'ont pas d'incidence significative sur l'obtention du bac, BarnetVerzat et Wolff [2003] et Gary-Bobo et al. [2006] trouvent que les filles sont d'autant plus défavorisées qu'elles ont de nombreux frères. Enfin, les derniers-nés dans les fratries s'en sortent relativement moins bien (Gary-Bobo et al. [2006]) ${ }^{8}$.

Au total, cette synthèse met en évidence les principaux facteurs qui expliquent la réussite scolaire en France, et nous allons chercher à prendre en compte ces différentes variables de contrôle dans notre analyse économétrique. Il n'en demeure pas moins que l'hétérogénéité intra-familiale de l'éducation apparaît peu documentée à ce jour ${ }^{9}$.

\section{DESCRIPTION DES DONNÉES}

Pour étudier l'hétérogénéité intra-familiale d'éducation en France, la principale contrainte en termes de données est liée à la nécessité de disposer d'informations sur le niveau d'éducation de chacun des frères et sœurs. Il s'agit d'une véritable difficulté au regard de la construction des enquêtes statistiques en France, qui privilégient usuellement le point de vue d'un enquêté et non celui d'une fratrie. Dans le cas général, le chef de ménage qui est interrogé n'apporte aucune précision sur la situation de ses propres frères et sœurs car il lui est seulement demandé de décrire les caractéristiques des enfants qui vivent chez lui au moment de l'enquête, dans le cadre du tableau de composition du ménage.

Dans cette étude, nous mobilisons les données de trois enquêtes réalisées par l'Insee et mises à disposition par le Centre Maurice Halbwachs : Actifs financiers 1992, Patrimoine 1998 et Patrimoine 2004. Ces sources statistiques présentent deux avantages principaux. En premier lieu, elles permettent de reconstituer des fratries complètes pour les enfants des enquêtés dans la mesure où leurs questionnaires comportent un module spécifique sur les enfants qui vivent hors du domicile parental à la date de l'enquête. De l'autre, elles s'appuient sur des échantillons de grande taille, entre 9500 et 10000 personnes de référence par enquête. Pour construire nos échantillons, nous procédons de la façon suivante.

Tout d'abord, nous construisons un fichier « parents » qui inclut les principales caractéristiques du chef de famille et de son éventuel conjoint. Ensuite, nous déterminons un fichier " enfants » pour lequel chaque enfant, quel que soit son lieu de résidence (au domicile de ses parents ou dans un logement indépendant), correspond à une observation. Pour chaque enfant, le chef de famille précise notamment leur sexe et leur âge, ce qui permet d'obtenir une caractérisation complète de la fratrie. Enfin, nous assemblons ces fichiers « parents » et « enfants » pour obtenir un échantillon apparié " parents-enfants ». Ainsi, une famille qui comprend $\mathrm{N}$ enfants contribue pour $\mathrm{N}$ observations à ce nouvel

8. Fabre et Moullet [2004] trouvent une corrélation négative entre le rang dans la fratrie et la durée de scolarité, mais le nombre de frères et sœurs n'est pas pris en compte dans leur régression. Ceci pose une difficulté pour l'interprétation, compte tenu de la corrélation mécanique entre la taille de la fratrie et le rang de naissance.

9. Comparant l'obtention du bac pour deux enfants d'un même ménage, Duée [2005] note que ces derniers ont le même résultat (obtention versus non-obtention) dans trois quarts des cas. Ceci suggère que l'hétérogénéité entre frères et sœurs reste limitée pour ce niveau de diplôme. 
échantillon. Cette procédure permet de disposer, pour chaque famille, à la fois des caractéristiques des parents et de chacun de leurs enfants.

Pour notre étude, nous avons choisi d'inclure les familles monoparentales et les familles recomposées. Des événements familiaux tels qu'un divorce ou un veuvage ont de fortes chances d'affecter les trajectoires scolaires des enfants. Une difficulté dans les enquêtes retenues vient de ce que la filiation exacte entre l'enfant et le chef de ménage enquêté ainsi que son éventuel conjoint n'est connue que pour l'enquête 2004. Nous savons alors si chaque enfant est un enfant du couple, de la personne de référence seule, ou du conjoint seul. Par définition, dans ce dernier cas, les enfants concernés ne sont pas des frères et sœurs des enfants de la personne de référence et ils sont donc exclus de l'échantillon apparié. En revanche, pour les enquêtes 1992 et 1998, le lien de filiation n'est pas connu pour les enfants vivant chez leurs parents (mais il l'est pour les enfants ayant quitté le domicile parental). Pour reconstituer des fratries, nous sommes donc amenés à faire l'hypothèse que les enfants vivant au domicile parental sont tous frères et sœurs dans les enquêtes 1992 et $1998^{10}$.

La variable que nous cherchons à expliquer est le niveau d'éducation de chaque enfant. Les trois enquêtes ne sont pas exactement symétriques en ce qui concerne l'information recueillie. Pour les enfants hors domicile, quatre modalités sont proposées pour le diplôme le plus élevé dans l'enquête 1992 : aucun diplôme, inférieur au bac, bac, supérieur au bac. Pour les enquêtes 1998 et 2004, deux modalités sont proposées pour les diplômes supérieurs au bac : bac +2 et supérieur à bac $+2^{11}$. Nous construisons donc une variable de diplôme ordonnée suivant cinq catégories (aucun diplôme, inférieur au bac, bac, bac +2 , supérieur à bac +2 ) pour ces deux enquêtes. Enfin, nous savons si les enfants sont scolarisés ou non à la date de l'enquête, à l'exception des enfants habitant chez leurs parents pour l'enquête 2004 .

Nous appliquons les trois sélections suivantes à l'échantillon apparié parentsenfants. Tout d'abord, nous éliminons les enfants âgés de moins 24 ans. Les enfants les plus jeunes sont très nombreux à être scolarisés à la date de l'enquête et ils n'ont pas encore achevé leurs études, si bien que leur diplôme final n'est pas encore connu. Inclure les enfants encore scolarisés donnerait lieu à un biais de sélection dans la mesure où il est vraisemblable que ceux qui sortent plus tôt du système scolaire viennent de milieux scolaires moins favorisés. Ensuite, nous supprimons de l'échantillon les mères dont l'âge est inférieur à 45 ans, dans la mesure où celles-ci peuvent ne pas avoir totalement complété leur descendance $^{12}$. Enfin, dans un souci de cohérence face à de possibles erreurs de mesure, nous avons exclu les enfants pour lesquels l'âge du père à la naissance ou l'âge de la mère à la naissance était inférieur à 14 ans.

10. Afin d'avoir une idée du biais engendré par cette hypothèse, nous avons comparé les deux scénarios suivants pour l'enquête 2004 : celui où la filiation est connue à la fois pour les enfants au domicile et hors domicile, et celui où la filiation des enfants au domicile avec le chef de ménage et son éventuel conjoint est (fictivement) supposée inconnue. Nous obtenons des résultats très similaires dans les deux cas.

11. L'absence d'informations sur l'éducation des enfants vivant chez leurs parents engendre un biais de sélection dans la mesure où l'âge de décohabitation est lié à l'âge de fin d'études et au diplôme. Pour les enfants qui vivent chez leurs parents, nous n'avons aucune information sur leur niveau d'éducation dans l'enquête 2004.

12. Nous avons également choisi d'exclure les ménages dont le chef de famille était inférieur à 45 ans, ce qui conduit à la suppression de 45 observations supplémentaires. 
Après élimination des observations manquantes, notre échantillon comprend 27197 paires parents-enfants, correspondant à 11694 familles : 8213 enfants pour 1992 (3 468 familles), 9684 enfants pour 1998 (4 099 familles) et 9300 enfants pour 2004 (4 127 familles). Le recours à trois enquêtes espacées dans le temps permet d'avoir de la profondeur dans la dimension temporelle pour notre étude : $15,3 \%$ des enfants sont nés avant $1950,30,3 \%$ entre 1950 et $1959,37,4 \%$ entre 1960 et 1969 , et $17,1 \%$ à partir de 1970 . L'âge moyen des enfants sélectionnés est de 38,3 ans.

La figure 1 décrit l'évolution du niveau de diplôme des enfants par cohorte de naissance, les deux catégories supérieures de diplôme (bac +2 et supérieur à bac +2 ) étant ici agrégées ${ }^{13}$. Les données mettent en évidence un premier fait stylisé, à savoir un accroissement continu du niveau de diplôme obtenu par ces générations sur la période, avec en particulier une accélération au niveau postbac sur la fin de la période. La proportion d'enfants avec un diplôme inférieur au bac est ainsi passée de 74,5\% pour ceux nés avant 1940 à $27,9 \%$ pour ceux nés après 1970 (elle est de 49,9\% pour ceux nés entre 1960 et 1964). Dans le même temps, la proportion de diplômés du supérieur est passée de 16,8 \% pour ceux nés avant 1940 à 35,6\% pour les cohortes nées entre 1960 et 1964, 45,7 \% entre 1965 et 1969, et 55,3\% pour celles nées après 1970 .

Un second fait stylisé porte sur la déformation de la structure des diplômes suivant le sexe des enfants au cours de la période. Les garçons nés avant 1950 sont en moyenne plus diplômés que les filles. Par exemple, 18,5 \% des garçons nés avant 1940 sont diplômés du supérieur contre 15,2 \% pour les filles. Les écarts s'inversent pour les générations nées après 1950. La proportion de filles diplômées du supérieur excède de 2,7 points celle des garçons pour les cohortes nées entre 1950 et 1954 (27,2\% au lieu de $25 \%$ ). L'écart est de 7,2 points pour celles nées entre 1960 et 1964 (39,2\% au lieu de $32 \%$ ) et même 11,2 points pour les cohortes nées à partir de 1970 (60,6 \% au lieu de 49,4\%).

Pour expliquer le diplôme des enfants, nous retenons les variables suivantes : le sexe, des indicatrices relatives aux cohortes de naissance, le nombre de frères et sœurs, le nombre de sœurs (à taille donnée de la fratrie) et le rang dans la fratrie en ce qui concerne les enfants ; l'âge de la personne de référence à la naissance de l'enfant, l'appartenance à une famille nucléaire, monoparentale ou recomposée $^{14}$, le niveau de diplôme de la personne de référence, et enfin une indicatrice de versement de transferts financiers des parents aux enfants (au niveau famille). Ce critère renvoie implicitement à la distinction entre des familles dites riches suivant la classification de Becker et Tomes [1986], pour lesquelles les parents procèdent à un investissement optimal dans le capital humain de leurs enfants et leur versent en complément de l'argent, et des familles dites pauvres contraintes par la liquidité et pour lesquelles aucun transfert financier n'est observé ${ }^{15}$. Les différentes variables explicatives retenues sont décrites dans le tableau 1.

13. La distinction entre ces deux niveaux de diplôme n'est en effet pas possible pour l'enquête 1992.

14. Les familles monoparentales correspondent ici à des familles avec un seul parent dans le tableau de composition de ménage, alors que les familles recomposées sont des familles où il y a des enfants ayant une filiation avec la personne de référence seulement.

15. Les transferts pris en compte ici sont des aides régulières ou ponctuelles, des dons et des donations. Les prêts sont en revanche exclus, dans la mesure où ils doivent a priori donner lieu à un remboursement de la part des enfants. Une limite de cette variable tient au fait que la richesse varie dans le temps, mais celle-ci n'est observée qu'au moment de l'enquête. 
Figure 1. Distribution de l'éducation des enfants, par cohorte de naissance des enfants

\section{A. Garçons et filles}

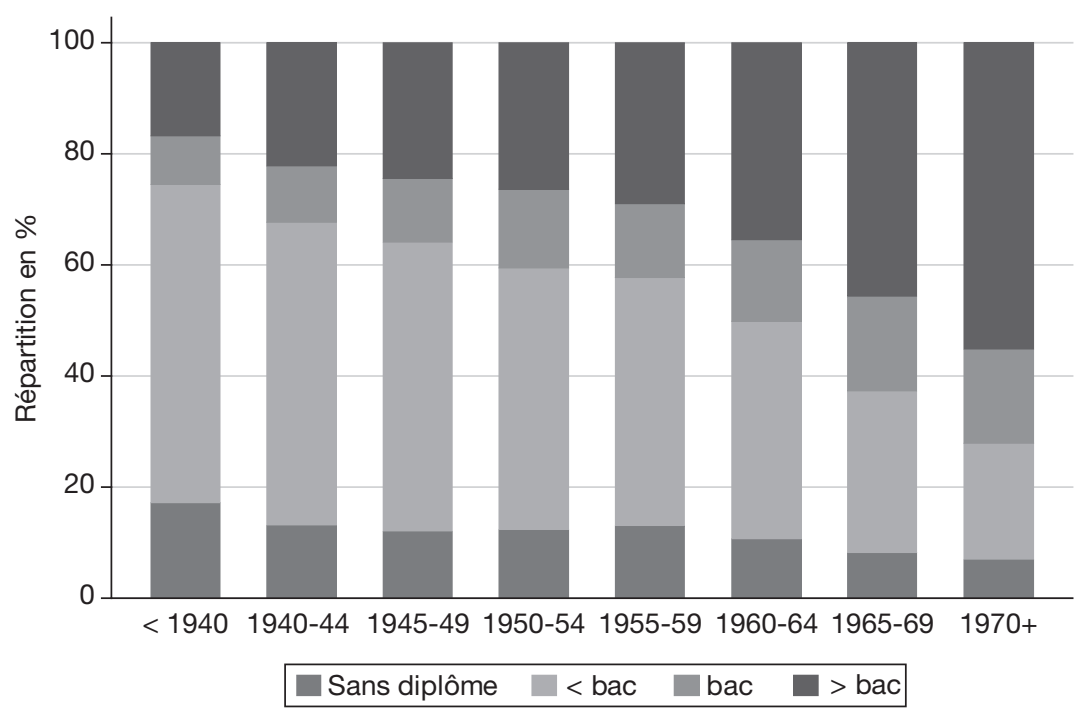

B. Par sexe

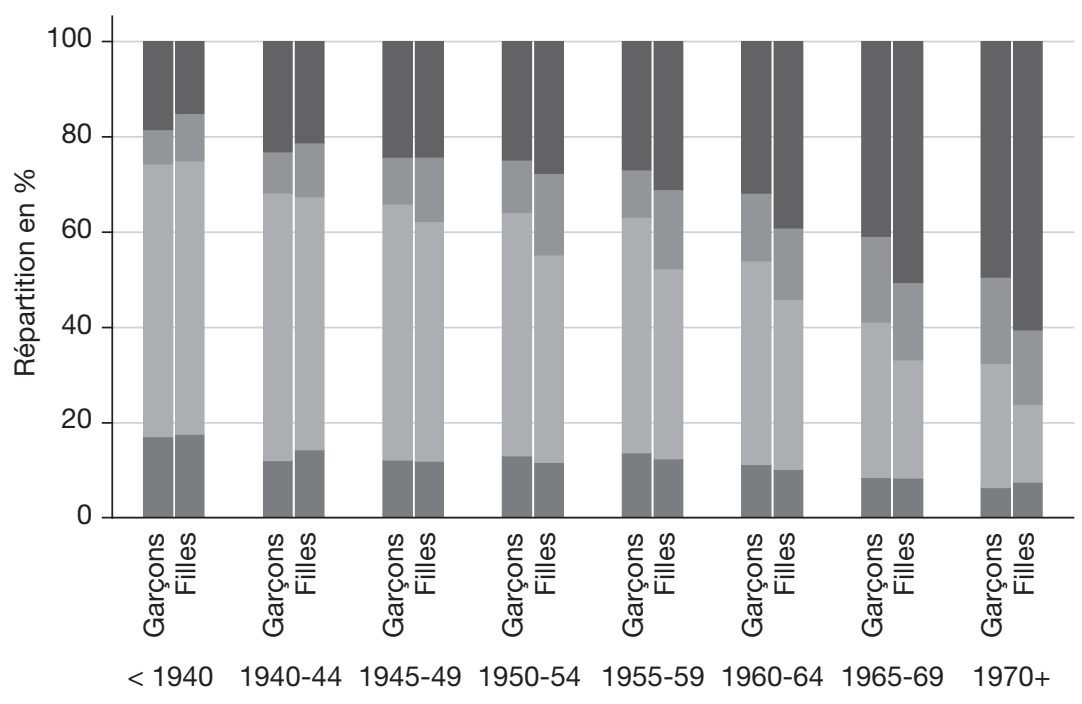

Sans diplôme $\quad<$ bac $\quad$ bac $\quad$ > bac

Sources : enquête Actifs financiers 1992, enquêtes Patrimoine 1998 et 2004. 
Tableau 1. Description de l'échantillon apparié parents-enfants

\begin{tabular}{|c|c|c|c|c|}
\hline \multicolumn{2}{|c|}{ Variables } & Garçons & Filles & Ensemble \\
\hline \multicolumn{5}{|c|}{ Caractéristiques des enfants } \\
\hline \multicolumn{2}{|l|}{ Sexe féminin } & 0,000 & 1,000 & 0,500 \\
\hline \multicolumn{2}{|l|}{ Âge } & 38,199 & 38,424 & 38,311 \\
\hline \multirow[t]{8}{*}{ Cohorte } & $<1945$ & 0,058 & 0,064 & 0,061 \\
\hline & 1945-1949 & 0,090 & 0,093 & 0,092 \\
\hline & $1950-1954$ & 0,136 & 0,134 & 0,135 \\
\hline & 1955-1959 & 0,167 & 0,168 & 0,168 \\
\hline & 1960-1964 & 0,196 & 0,191 & 0,194 \\
\hline & $1965-1969$ & 0,181 & 0,178 & 0,180 \\
\hline & $1970-1974$ & 0,124 & 0,122 & 0,123 \\
\hline & $1975+$ & 0,046 & 0,050 & 0,048 \\
\hline \multicolumn{2}{|c|}{ Nombre de frères et sœurs } & 2,430 & 2,478 & 2,454 \\
\hline \multicolumn{2}{|l|}{ Nombre de frères } & 1,223 & 1,226 & 1,225 \\
\hline \multicolumn{2}{|l|}{ Nombre de sœurs } & 1,207 & 1,251 & 1,229 \\
\hline \multicolumn{2}{|c|}{ Rang absolu dans la fratrie } & 2,127 & 2,135 & 2,131 \\
\hline \multirow[t]{4}{*}{ Diplôme } & Sans & 0,119 & 0,115 & 0,117 \\
\hline & $<$ bac & 0,469 & 0,405 & 0,437 \\
\hline & $\mathrm{Bac}$ & 0,120 & 0,150 & 0,135 \\
\hline & $>$ bac & 0,292 & 0,330 & 0,311 \\
\hline \multicolumn{5}{|c|}{ Caractéristiques du parent de référence } \\
\hline \multicolumn{2}{|c|}{ Âge du parent à la naissance de l'enfant } & 27,964 & 27,868 & 27,916 \\
\hline \multicolumn{2}{|l|}{ Famille nucléaire } & 0,617 & 0,601 & 0,609 \\
\hline \multicolumn{2}{|c|}{ Famille monoparentale } & 0,344 & 0,359 & 0,351 \\
\hline \multicolumn{2}{|l|}{ Famille recomposée } & 0,039 & 0,040 & 0,040 \\
\hline \multirow[t]{5}{*}{ Éducation du parent } & Sans & 0,285 & 0,293 & 0,289 \\
\hline & CEP & 0,306 & 0,301 & 0,303 \\
\hline & CAP-BEP-BEPC & 0,211 & 0,207 & 0,209 \\
\hline & $\mathrm{Bac}$ & 0,082 & 0,083 & 0,082 \\
\hline & $>$ bac & 0,117 & 0,116 & 0,116 \\
\hline \multicolumn{2}{|c|}{ Famille riche (transferts aux enfants) } & 0,469 & 0,469 & 0,469 \\
\hline \multicolumn{2}{|c|}{ Nombre d'observations } & 13605 & 13592 & 27197 \\
\hline
\end{tabular}

Source: enquête Actifs financiers 1992, enquêtes Patrimoine 1998 et 2004.

Dans une perspective dynamique, deux variables jouant un rôle majeur sur l'éducation des enfants se sont largement modifiées sur la période, à savoir la taille des fratries et le niveau d'éducation des parents. D'une part, comme le montre la figure 2, les générations du baby-boom qui sont nées entre 1945 et 1959 appartiennent à des fratries de taille plus importante. La baisse de la fécondité en France après 1960 est venue mécaniquement réduire le nombre moyen de frères et sœurs pour un enfant donné. D'autre part, les enfants issus des plus jeunes cohortes de naissance ont des parents plus souvent diplômés du supérieur, 
tandis que la proportion de ceux qui ont au plus le certificat d'études primaires s'est fortement réduite dans le temps (fig. 3). Ces évolutions ont pour conséquence un accroissement du niveau d'éducation moyen des enfants.

Figure 2. Évolution du nombre de frères et sœurs, par cohorte de naissance des enfants

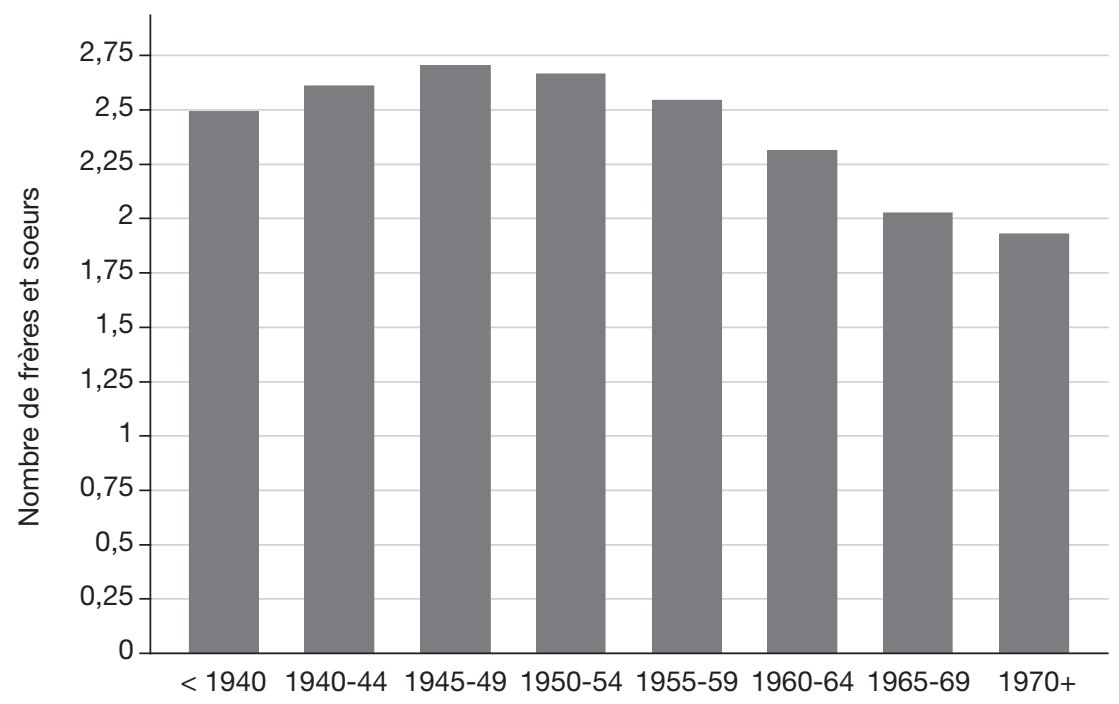

Source : enquête Actifs financiers 1992, enquêtes Patrimoine 1998 et 2004.

Figure 3. Distribution du niveau d'éducation du parent de référence, par cohorte de naissance des enfants

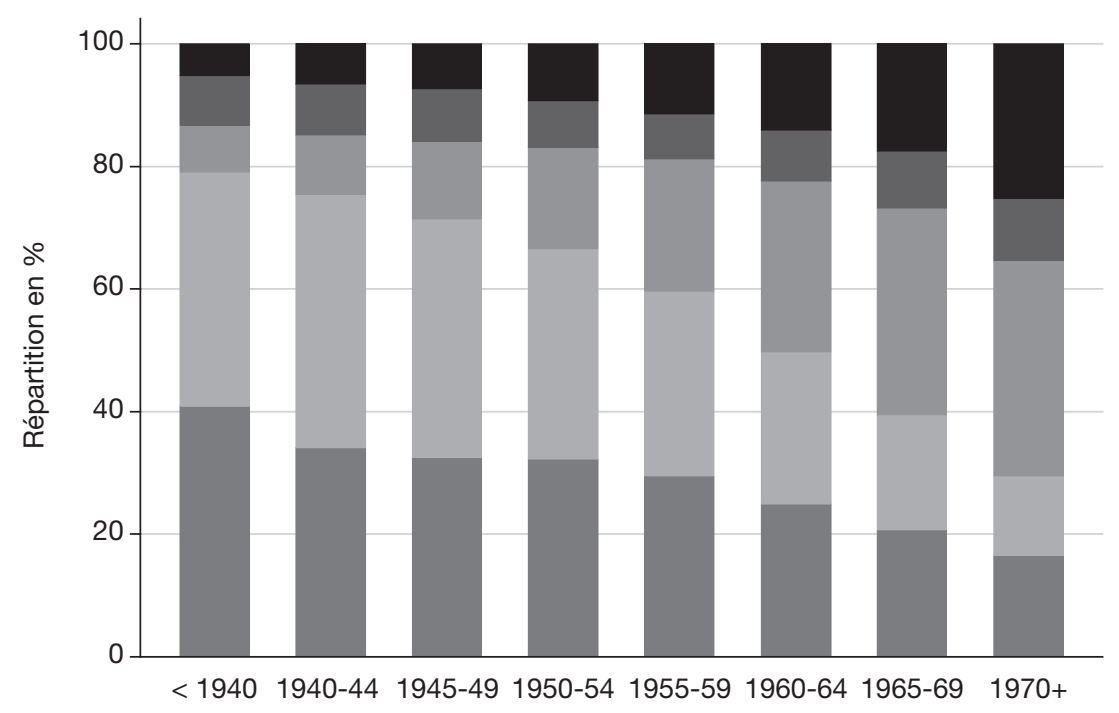

Source : enquête Actifs financiers 1992, enquêtes Patrimoine 1998 et 2004. 
Le tableau 2 apporte des premiers éléments sur le rôle joué par les facteurs explicatifs retenus sur le niveau éducatif des enfants. Les filles ont plus souvent le bac ou un diplôme supérieur que les garçons, et les plus diplômés sont en moyenne plus jeunes. Le niveau d'éducation décroît fortement avec le nombre de frères et sœurs et avec le rang dans la fratrie. En ce qui concerne les parents, l'âge moyen du parent de référence à la naissance tend à augmenter avec le diplôme (à partir du bac). Ce dernier est corrélé négativement avec l'appartenance à une famille monoparentale. L'effet du niveau d'éducation du parent de référence est très significatif. Les enfants ont plus souvent un diplôme du supérieur lorsque le parent de référence a obtenu le bac et surtout lorsque le parent est lui-même diplômé du supérieur. Enfin, la proportion de familles riches croît fortement avec les niveaux d'éducation des enfants.

Tableau 2. Description de l'échantillon apparié parents-enfants, par diplôme de l'enfant

\begin{tabular}{|c|c|c|c|c|c|}
\hline \multicolumn{2}{|c|}{ Variables } & Sans & $<$ bac & $\mathrm{Bac}$ & $>$ bac \\
\hline \multicolumn{6}{|c|}{ Caractéristiques des enfants } \\
\hline \multicolumn{2}{|l|}{ Sexe féminin } & 0,491 & 0,463 & 0,556 & 0,531 \\
\hline \multicolumn{2}{|l|}{ Âge } & 39,623 & 39,837 & 37,062 & 36,214 \\
\hline \multirow[t]{8}{*}{ Cohorte } & $<1945$ & 0,089 & 0,080 & 0,039 & 0,033 \\
\hline & $1945-1949$ & 0,103 & 0,114 & 0,068 & 0,066 \\
\hline & $1950-1954$ & 0,138 & 0,161 & 0,116 & 0,106 \\
\hline & $1955-1959$ & 0,176 & 0,181 & 0,176 & 0,142 \\
\hline & 1960-1964 & 0,215 & 0,198 & 0,190 & 0,181 \\
\hline & $1965-1969$ & 0,163 & 0,161 & 0,194 & 0,206 \\
\hline & 1970-1974 & 0,087 & 0,081 & 0,157 & 0,181 \\
\hline & $1975+$ & 0,028 & 0,023 & 0,060 & 0,085 \\
\hline \multicolumn{2}{|c|}{ Nombre de frères et sœurs } & 3,583 & 2,688 & 2,085 & 1,859 \\
\hline \multicolumn{2}{|l|}{ Nombre de frères } & 1,773 & 1,353 & 1,033 & 0,921 \\
\hline \multicolumn{2}{|l|}{ Nombre de sœurs } & 1,810 & 1,335 & 1,052 & 0,938 \\
\hline \multicolumn{2}{|l|}{ Rang dans la fratrie } & 2,675 & 2,243 & 1,991 & 1,830 \\
\hline \multicolumn{6}{|c|}{ Caractéristiques des parents } \\
\hline \multicolumn{2}{|c|}{ Âge du parent à la naissance de l'enfant } & 27,872 & 27,662 & 28,017 & 28,246 \\
\hline \multicolumn{2}{|l|}{ Famille nucléaire } & 0,479 & 0,581 & 0,623 & 0,692 \\
\hline \multicolumn{2}{|c|}{ Famille monoparentale } & 0,471 & 0,382 & 0,335 & 0,270 \\
\hline \multicolumn{2}{|l|}{ Famille recomposée } & 0,051 & 0,037 & 0,042 & 0,038 \\
\hline \multirow[t]{5}{*}{ Éducation du parent } & Sans & 0,608 & 0,359 & 0,204 & 0,108 \\
\hline & CEP & 0,245 & 0,385 & 0,315 & 0,206 \\
\hline & CAP-BEP-BEPC & 0,108 & 0,190 & 0,269 & 0,247 \\
\hline & $\mathrm{Bac}$ & 0,022 & 0,042 & 0,113 & 0,149 \\
\hline & $>$ bac & 0,018 & 0,024 & 0,099 & 0,290 \\
\hline \multicolumn{2}{|c|}{ Famille riche (transferts aux enfants) } & 0,302 & 0,391 & 0,476 & 0,639 \\
\hline \multicolumn{2}{|c|}{ Nombre d'observations } & 3187 & 11886 & 3667 & 8457 \\
\hline \multicolumn{6}{|c|}{ Source : enquête Actifs financiers 1992, enquêtes Patrimoine 1998 et 2004.} \\
\hline
\end{tabular}


Naturellement, les variables que nous prenons en considération ne doivent pas faire oublier que l'éducation n'est pas seulement un choix privé. En 2004, par exemple, les dépenses intérieures d'éducation représentaient 7,1\% du PIB, mais seulement $11,2 \%$ de cette dépense totale étaient le fait des ménages ${ }^{16}$. La dépense intérieure d'éducation a globalement augmenté sur la moyenne période : $6,4 \%$ du PIB en 1974, $7 \%$ en 1984 et 7,6 \% en 1997, pour ensuite se stabiliser autour de $7 \%$ (fig. 4). Dans les modèles estimés ici, les conditions différenciées rencontrées par les générations successives d'enfants sont capturées par des indicatrices relatives aux cohortes de naissance. Nous avons également pris en compte la région de résidence des parents ainsi que le fait de vivre dans une zone rurale afin de contrôler la disponibilité de l'offre scolaire et d'autres aspects culturels liés ${ }^{17}$.

Figure 4. Dépense moyenne d'éducation par élève

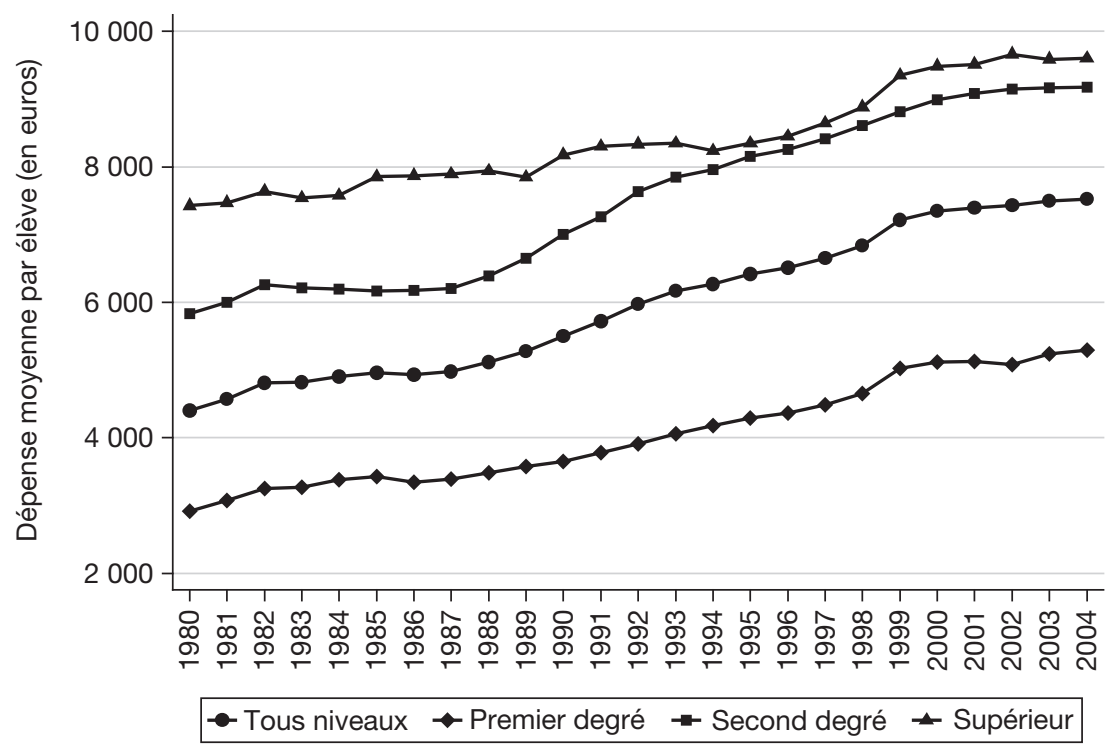

Il s'agit de dépenses en euros constants, au prix de 2008.

Source : données DEPP, Tableaux de l'économie française 2010, Insee.

\section{SPÉCIFICATION ÉCONOMÉTRIQUE}

Pour expliquer le niveau de diplôme d'un enfant $i\left(i=1, \ldots, \mathrm{N}_{j}\right)$ appartenant à une famille $j(j=1, \ldots, \mathrm{J})$ de taille $\mathrm{N}_{j}$, nous supposons qu'il existe une variable

16. Se reporter au document suivant: http://media.education.gouv.fr/file/32/1/2321.pdf.

17. Il s'agit toutefois de la résidence du parent de référence à la date de l'enquête, ce qui ne correspond pas nécessairement (en cas de mobilité géographique) au lieu où les enfants vivaient lors de leur formation initiale. Il n'existe toutefois pas d'information plus précise dans les enquêtes Actifs financiers et Patrimoine. 
latente (inobservée) $\mathrm{E}_{j i}^{*}$ qui mesure la propension à être éduqué. Celle-ci est modélisée sous la forme linéaire suivante :

$$
\mathrm{E}_{j i}^{*}=\mathrm{X}_{j i} \beta+\varepsilon_{j i}
$$

avec $\mathrm{X}_{j i}$ les facteurs explicatifs retenus, $\beta$ le vecteur de paramètres associés et $\varepsilon_{j i}$ une perturbation aléatoire. Nous supposons que le terme résiduel peut s'écrire comme la somme d'un effet spécifique $\delta_{j}$ propre à chaque famille et d'un pur terme aléatoire $\xi_{j i}$ :

$$
\varepsilon_{j i}=\delta_{j}+\xi_{j i}
$$

L'effet familial $\delta_{j}$ capture l'ensemble des caractéristiques parentales inobservées qui peuvent influencer l'investissement en capital humain pour tous les enfants de la famille (par exemple le degré d'altruisme parental ou encore les traits génétiques familiaux hérités) ainsi que les possibles erreurs de mesure spécifiques aux parents ${ }^{18}$. Les capacités intrinsèques aux enfants qui ne sont pas directement observables, telles que leurs aptitudes ou leur intérêt pour les études qui va conditionner leur réussite, sont prises en compte à travers le terme $\xi_{j i}$. Ce terme reflète également l'influence des caractéristiques inobservables des enfants ou des parents qui pousseraient ces derniers à favoriser, en matière d'éducation, un de leurs enfants plutôt qu'un autre.

Nous supposons que $\delta_{j}$ et $\xi_{j i}$ suivent des lois normales telles que $\delta_{j} \sim \mathrm{N}\left(0 ; \sigma_{\delta}^{2}\right)$ et $\xi_{j i} \sim \mathrm{N}(0 ; 1)$, la variance du terme résiduel $\xi_{j i}$ étant donc normalisée à 1 . Les termes d'erreur $\delta_{j}$ et $\xi_{j i}$ sont, par ailleurs, supposés indépendants et non corrélés avec les variables explicatives $\mathrm{X}_{j i}$ retenues dans la régression. Par définition, le niveau latent $\mathrm{E}_{j i}^{*}$ demeure inconnu. Seul le diplôme obtenu $\mathrm{E}_{j i}$ est connu. Nous posons $\mathrm{E}_{j i}=k$ lorsque l'enfant a le diplôme $k$. Le lien entre le niveau observé d'éducation et la variable latente correspondante est donné par la condition $\mathrm{E}_{j i}=k$ lorsque $\mu_{k-1}<\mathrm{E}_{j i}^{*} \leqslant \mu_{k}$. Le modèle correspondant est un modèle Probit ordonné et les seuils $\mu_{k}$ sont estimés simultanément avec les coefficients $\beta$. Sachant que $\operatorname{Pr}\left(\mathrm{E}_{j i}=k\right)=\operatorname{Pr}\left(\mu_{k-1}<\mathrm{E}_{j i}^{*} \geqslant \mu_{k}\right)$, la probabilité que l'enfant $i$ né dans la famille $j$ atteigne le niveau d'éducation $k$, conditionnellement à l'effet familial $\delta_{j}$, s'écrit sous la forme :

$$
\operatorname{Pr}\left(\mathrm{E}_{j i}=k \mid \delta_{j}\right)=\Phi\left(\mu_{k}-\mathrm{X}_{j i} \beta-\delta_{j}\right)-\Phi\left(\mu_{k-1}-\mathrm{X}_{j i} \beta-\delta_{j}\right)
$$

avec $\Phi$ la fonction de répartition de la loi normale centrée réduite. Pour une fratrie donnée, nous en déduisons :

$$
\begin{aligned}
& \operatorname{Pr}\left(\mathrm{E}_{j 1}, \ldots, \mathrm{E}_{j N_{j}}\right)= \\
& \int_{-\infty}^{+\infty} \prod_{i=1}^{\mathrm{N}_{j}}\left[\Phi\left(\mu_{k}-\mathrm{X}_{j i} \beta-\delta_{j}\right)-\Phi\left(\mu_{k-1}-\mathrm{X}_{j i} \beta-\delta_{j}\right)\right] \phi\left(\delta_{j}\right) d \delta_{j}
\end{aligned}
$$

avec $\varphi$ la fonction de densité de la loi normale centrée réduite. La spécification (4) définit un modèle Probit ordonné à effets aléatoires, qui peut être estimé

18. Par construction, ce sont les parents dans l'enquête qui décrivent la situation de chacun de leurs enfants. Il se peut, par exemple, que, d'une famille à l'autre, les parents n'aient pas la même appréciation de ce que représente le diplôme de leurs enfants, certains pouvant se référer à des niveaux atteints et d'autres à des diplômes effectivement obtenus. Si l'appréciation subjective des parents s'applique pour tous les frères et sœurs, alors ces différences vont être capturées par cet effet spécifique familial. 
facilement par l'application de techniques de quadrature gaussienne (Butler et Moffit [1982] ; Frechette [2001]).

Une difficulté dans notre étude vient de ce que nous n'avons pas exactement la même information sur les diplômes dans l'enquête Actifs financiers 1992 et les enquêtes Patrimoine 1998 et 2004 . Soit $\mathrm{E}_{j i}=1$ quand l'enfant n'a aucun diplôme, $\mathrm{E}_{j i}=2$ quand il a un diplôme inférieur au bac, $\mathrm{E}_{j i}=3$ quand il a le bac, $\mathrm{E}_{j i}=4$ quand il a un diplôme de type bac +2 et $\mathrm{E}_{j i}=5$ quand il a un diplôme supérieur à bac +2 . Dans notre échantillon, la distinction entre $\mathrm{E}_{j i}=4$ et $\mathrm{E}_{j i}=5$ n'est pas possible pour les enfants hors domicile dans l'enquête 1992. Dans ce dernier cas, nous savons seulement si les enfants ont un diplôme supérieur au bac ou non. Dans l'affirmative, nous avons $\mathrm{E}_{j i}=4$ ou $\mathrm{E}_{j i}=5$, ce que nous pouvons encore écrire $\mathrm{E}_{j i} \geqslant 4$.

La vraisemblance conditionnelle à $\delta$ n'est pas exactement la même pour les familles observées en 1992. Soit $\Theta_{j i}$ une variable muette qui prend pour valeur 0 lorsque l'enfant est interrogé en 1992, ne vit plus chez leurs parents et est diplômés du supérieur, tandis que $\Theta_{j i}$ est égal à 1 dans le cas contraire. Pour les observations telles que $\Theta_{j i}=0$, lorsque le niveau de diplôme est tel que $\mathrm{E}_{j i} \geqslant k$, la probabilité $\operatorname{Pr}\left(\mathrm{E}_{j i} \geqslant k \mid \delta_{j}\right)$ peut s'écrire :

$$
\operatorname{Pr}\left(\mathrm{E}_{j i} \geqslant k \mid \delta_{j}\right)=\operatorname{Pr}\left(\mathrm{E}_{j i}^{*}>\mu_{k-1} \mid \delta_{j}\right)^{19} .
$$

Dès lors, il vient :

$$
\operatorname{Pr}\left(\mathrm{E}_{j i} \geqslant k \mid \delta_{j}\right)=1-\Phi\left(\mu_{k-1}-\mathrm{X}_{j i} \beta-\delta_{j}\right)
$$

La contribution à la vraisemblance est alors donnée par :

$$
\begin{aligned}
& \operatorname{Pr}\left(\mathrm{E}_{j 1}, \ldots, \mathrm{E}_{j \mathrm{~N}_{j}}\right)= \\
& \int_{-\infty}^{+\infty} \prod_{i=1}^{\mathrm{N}_{j}}\left\{\begin{array}{c}
\Theta_{j i} *\left[\Phi\left(\mu_{k}-\mathrm{X}_{j i} \beta-\delta_{j}\right)-\Phi\left(\mu_{k-1}-\mathrm{X}_{j i} \beta-\delta_{j}\right)\right] \\
+\left(1-\Theta_{j i}\right) *\left[1-\Phi\left(\mu_{k-1}-\mathrm{X}_{j i} \beta-\delta_{j}\right)\right]
\end{array}\right\} \Phi\left(\delta_{j}\right) d \delta_{j} .
\end{aligned}
$$

Ce modèle nous permet ainsi de mesurer l'hétérogénéité d'éducation à la fois entre les familles (dimension inter) et au sein des familles (dimension intra), suivant la méthodologie basée sur une décomposition de variance proposée par Picard et Wolff [2010].

Nous calculons tout d'abord la valeur prédite $\hat{E}_{j i}^{*}=X_{j i} \hat{\beta}$ associée au niveau d'éducation latent pour chaque enfant. Nous en déduisons alors la propension moyenne à être éduquée $\hat{\mathrm{E}}_{j}^{*}$ pour chaque famille et l'écart individuel par rapport à ce niveau moyen $\hat{\mathrm{E}}_{j i}^{*}-\hat{\mathrm{E}}_{j}^{*}$. De ces calculs sont obtenues la variance expliquée inter $\mathrm{V}\left(\hat{\mathrm{E}}_{j}^{*}\right)$ qui mesure les écarts moyens d'éducation entre les familles et la variance expliquée intra $\mathrm{V}\left(\hat{\mathrm{E}}_{j i}^{*}-\hat{\mathrm{E}}_{j}^{*}\right)$ qui mesure le poids des variations d'éducation autour de ce niveau moyen pour chaque famille. Cette décomposition est complétée par les variances inexpliquées obtenues à partir des termes résiduels, respectivement $\hat{\sigma}_{\delta}^{2}$ pour la dimension inter et $\hat{\sigma}_{\xi}^{2}=1$ pour la dimension intra.

19. Nous supposons ici l'exogénéité de la variable $\Theta_{j i}$. Il est toutefois vraisemblable que les enfants ont d'autant plus de chances d'être fortement diplômés (donc au-delà du bac +2 ) que leurs parents sont eux-mêmes très diplômés. 


\section{RÉSULTATS ÉCONOMÉTRIQUES}

\section{Une mesure de l'hétérogénéité d'éducation en France}

Nous estimons tout d'abord un modèle ordonné à effets aléatoires pour les enfants âgés de 24 ans et plus sur la base d'une variable d'éducation ordonnée avec les quatre niveaux suivants : sans diplôme, inférieur au bac, bac, supérieur au bac.

D'après les résultats présentés dans la colonne $1 \mathrm{du}$ tableau 3 , le niveau d'éducation est plus important pour les filles que pour les garçons (au seuil de $1 \%$ ). L'éducation est également fortement corrélée avec la cohorte de naissance de l'enfant. Les enfants ont en moyenne des diplômes d'autant plus importants qu'ils appartiennent à des cohortes récentes. Des tests plus précis montrent que l'éducation n'est pas différente pour les cohortes nées entre 1950 et 1964, tandis que les coefficients pour les cohortes nées entre 1965 et 1969, celles nées entre 1970 et 1974 et celles nées à partir de 1975 sont de plus en plus élevés. Cette croissance régulière de l'éducation peut trouver plusieurs origines : effort d'investissement accru de la part des familles, davantage de fonds publics consacrés à l'éducation, mais aussi de possibles modifications dans les modalités d'accès aux diplômes par le fait d'une moindre sélectivité (Magnac et Thesmar [2002]).

Tableau 3. Modèles Probit ordonnés à effets aléatoires expliquant l'éducation des enfants

\begin{tabular}{|c|c|c|c|c|c|}
\hline \multirow{2}{*}{\multicolumn{2}{|c|}{ Variables }} & \multirow{2}{*}{$\begin{array}{c}4 \text { niveaux } \\
\text { d'éducation }\end{array}$} & \multicolumn{3}{|c|}{5 niveaux d'éducation } \\
\hline & & & \multirow[t]{2}{*}{ Ensemble } & \multicolumn{2}{|c|}{ Avec effets croisés } \\
\hline \multicolumn{4}{|c|}{ Caractéristiques des enfants } & & $\begin{array}{l}\text { (x sexe } \\
\text { féminin) }\end{array}$ \\
\hline Sexe féminin & & $\begin{array}{c}0,209 * * * \\
(10,97)\end{array}$ & $\begin{array}{c}0,191 * * * \\
(10,41)\end{array}$ & $\begin{array}{r}-0,102 \\
(-0,82)\end{array}$ & \\
\hline \multirow[t]{7}{*}{$\begin{array}{l}\text { Cohorte } \\
\text { (réf. : < 1945) }\end{array}$} & 1945-1949 & $\begin{array}{c}0,128 * * * \\
(2,67)\end{array}$ & $\begin{array}{c}0,140 * * * \\
(2,97)\end{array}$ & $\begin{array}{l}0,106 \\
(1,60)\end{array}$ & $\begin{array}{l}0,063 \\
(0,71)\end{array}$ \\
\hline & $1950-1954$ & $\begin{array}{c}0,226 * * * \\
(4,73)\end{array}$ & $\begin{array}{c}0,226 * * * \\
(4,85)\end{array}$ & $\begin{array}{c}0,117^{*} \\
(1,84)\end{array}$ & $\begin{array}{c}0,207 * * \\
(2,48)\end{array}$ \\
\hline & $1955-1959$ & $\begin{array}{c}0,259 * * * \\
(5,35)\end{array}$ & $\begin{array}{c}0,254 * * * \\
(5,35)\end{array}$ & $\begin{array}{l}0,067 \\
(1,05)\end{array}$ & $\begin{array}{c}0,360 * * * \\
(4,40)\end{array}$ \\
\hline & $1960-1964$ & $\begin{array}{c}0,222 * * * \\
(4,48)\end{array}$ & $\begin{array}{c}0,215^{* * *} \\
(4,45)\end{array}$ & $\begin{array}{l}0,053 \\
(0,83)\end{array}$ & $\begin{array}{c}0,310 * * * \\
(3,79)\end{array}$ \\
\hline & 1965-1969 & $\begin{array}{c}0,398 * * * \\
(7,73)\end{array}$ & $\begin{array}{c}0,369 * * * \\
(7,36)\end{array}$ & $\begin{array}{c}0,177 * * * \\
(2,69)\end{array}$ & $\begin{array}{c}0,376^{* * *} \\
(4,52)\end{array}$ \\
\hline & $1970-1974$ & $\begin{array}{c}0,673 * * * \\
(12,24)\end{array}$ & $\begin{array}{c}0,603 * * * \\
(11,35)\end{array}$ & $\begin{array}{c}0,421 * * * \\
(6,06)\end{array}$ & $\begin{array}{c}0,352 * * * \\
(4,00)\end{array}$ \\
\hline & $1975+$ & $\begin{array}{c}0,823 * * * \\
(12,18)\end{array}$ & $\begin{array}{c}0,752 * * * \\
(11,73)\end{array}$ & $\begin{array}{c}0,509 * * * \\
(5,98)\end{array}$ & $\begin{array}{c}0,476 * * * \\
(4,37)\end{array}$ \\
\hline \multicolumn{2}{|c|}{ Nombre de frères et sœurs } & $\begin{array}{c}-0,190 * * * \\
(-17,06)\end{array}$ & $\begin{array}{c}-0,184 * * * \\
(-16,94)\end{array}$ & $\begin{array}{c}-0,163 * * * \\
(-11,90)\end{array}$ & $\begin{array}{c}-0,031 * * \\
(-2,09)\end{array}$ \\
\hline
\end{tabular}


Nathalie Picard, François-Charles Wolff

\begin{tabular}{l|c|c|c|c}
\hline \multirow{2}{*}{ Variables } & \multirow{2}{*}{$\begin{array}{c}\text { 4 niveaux } \\
\text { d'éducation }\end{array}$} & \multicolumn{2}{|c}{5 niveaux d'éducation } \\
\cline { 3 - 5 } & & Ensemble & \multicolumn{2}{|c}{ Avec effets croisés } \\
\hline Nombre de sœurs & $\begin{array}{c}0,054^{* * *} \\
(3,45)\end{array}$ & $\begin{array}{c}0,051^{* * *} \\
(3,42)\end{array}$ & $\begin{array}{c}0,038^{* *} \\
(1,98)\end{array}$ & $\begin{array}{c}0,004 \\
(0,19)\end{array}$ \\
\hline Rang normalisé & $\begin{array}{c}0,359 * * * \\
(-12,79)\end{array}$ & $\begin{array}{c}-0,343 * * * \\
(-12,73)\end{array}$ & $\begin{array}{c}-0,327 * * * \\
(-8,68)\end{array}$ & $\begin{array}{c}-0,037 \\
(-0,71)\end{array}$ \\
\hline
\end{tabular}

Caractéristiques de la personne de référence

\begin{tabular}{|c|c|c|c|c|c|}
\hline \multicolumn{2}{|c|}{$\begin{array}{l}\text { Âge du parent à la naissance } \\
\text { de l'enfant }\end{array}$} & $\begin{array}{c}0,030 * * * \\
(12,00)\end{array}$ & $\begin{array}{l}0,029 * * * \\
(12,13)\end{array}$ & $\begin{array}{l}0,027 * * * \\
(8,90)\end{array}$ & $\begin{array}{l}0,004 \\
(1,09)\end{array}$ \\
\hline \multicolumn{2}{|c|}{ Famille monoparentale } & $\begin{array}{c}-0,138 * * * \\
(-4,93)\end{array}$ & $\begin{array}{c}-0,122 * * * \\
(-4,52)\end{array}$ & $\begin{array}{c}-0,114 * * * \\
(-3,46)\end{array}$ & $\begin{array}{l}-0,014 \\
(-0,37)\end{array}$ \\
\hline \multicolumn{2}{|c|}{ Famille recomposée } & $\begin{array}{c}-0,485 * * * \\
(-8,31)\end{array}$ & $\begin{array}{c}-0,454 * * * \\
(-8,07)\end{array}$ & $\begin{array}{c}-0,413 * * * \\
(-5,78)\end{array}$ & $\begin{array}{l}-0,085 \\
(-0,98)\end{array}$ \\
\hline \multirow[t]{4}{*}{$\begin{array}{l}\text { Éducation du p } \\
\text { (réf. : sans) }\end{array}$} & CEP & $\begin{array}{l}0,601 * * * \\
(18,20)\end{array}$ & $\begin{array}{l}0,594 * * * \\
(18,42)\end{array}$ & $\begin{array}{l}0,591 * * * \\
(15,20)\end{array}$ & $\begin{array}{l}0,011 \\
(0,25)\end{array}$ \\
\hline & CAP-BEP-BEPC & $\begin{array}{l}0,974 * * * \\
(26,05)\end{array}$ & $\begin{array}{l}0,953 * * * \\
(26,28)\end{array}$ & $\begin{array}{l}0,908 * * * \\
(20,65)\end{array}$ & $\begin{array}{c}0,100 * * \\
(1,99)\end{array}$ \\
\hline & $\mathrm{Bac}$ & $\begin{array}{c}1,702 * * * \\
(33,06)\end{array}$ & $\begin{array}{c}1,651 * * * \\
(33,73)\end{array}$ & $\begin{array}{c}1,698 * * * \\
(28,43)\end{array}$ & $\begin{array}{r}-0,087 \\
(-1,28)\end{array}$ \\
\hline & $>$ bac & $\begin{array}{c}2,310 * * * \\
(44,54)\end{array}$ & $\begin{array}{c}2,295 * * * \\
(47,38)\end{array}$ & $\begin{array}{c}2,363 * * * \\
(40,22)\end{array}$ & $\begin{array}{r}-0,122^{*} \\
(-1,83)\end{array}$ \\
\hline \multicolumn{2}{|c|}{$\begin{array}{l}\text { Famille riche (transferts } \\
\text { aux enfants) }\end{array}$} & $\begin{array}{c}0,393 * * * \\
(15,23)\end{array}$ & $\begin{array}{l}0,402 * * * \\
(16,12)\end{array}$ & $\begin{array}{c}0,388 * * * \\
(12,80)\end{array}$ & $\begin{array}{l}0,027 \\
(0,79)\end{array}$ \\
\hline \multirow[t]{3}{*}{ Variance inter } & Expliquée & $\begin{array}{c}1,074 \\
(35,6 \%)\end{array}$ & $\begin{array}{c}1,034 \\
(35,1 \%)\end{array}$ & \multicolumn{2}{|c|}{$1,042(35,1 \%)$} \\
\hline & Inexpliquée & $\begin{array}{c}0,933 \\
(30,9 \%)\end{array}$ & $\begin{array}{c}0,902 \\
(30,6 \%)\end{array}$ & \multicolumn{2}{|c|}{$0,906(30,6 \%)$} \\
\hline & Total & $\begin{array}{c}2,007 \\
(66,5 \%)\end{array}$ & $\begin{array}{c}1,936 \\
(65,7 \%)\end{array}$ & \multicolumn{2}{|c|}{$1,948(65,7 \%)$} \\
\hline \multirow[t]{3}{*}{ Variance intra } & Expliquée & $\begin{array}{c}0,014 \\
(0,4 \%)\end{array}$ & $\begin{array}{c}0,012 \\
(0,4 \%)\end{array}$ & \multicolumn{2}{|c|}{$0,016(0,5 \%)$} \\
\hline & Inexpliquée & $\begin{array}{c}1,000 \\
(33,1 \%)\end{array}$ & $\begin{array}{c}1,000 \\
(33,9 \%)\end{array}$ & \multicolumn{2}{|c|}{$1,000(33,7 \%)$} \\
\hline & Total & $\begin{array}{c}1,014 \\
(33,5 \%)\end{array}$ & $\begin{array}{c}1,012 \\
(34,3 \%)\end{array}$ & \multicolumn{2}{|c|}{$1,016(34,3 \%)$} \\
\hline \multicolumn{2}{|c|}{ Nombre d'observations } & 27197 & 27197 & \multicolumn{2}{|c|}{27197} \\
\hline \multicolumn{2}{|c|}{ Nombre de familles } & 11694 & 11694 & \multicolumn{2}{|c|}{11694} \\
\hline \multicolumn{2}{|c|}{ Log vraisemblance } & $-27410,5$ & $-31292,6$ & \multicolumn{2}{|c|}{$-31238,2$} \\
\hline
\end{tabular}

Les régressions estimées sont des modèles Probit ordonnés à effets aléatoires. Les seuils de significativité retenus sont respectivement de $1 \%(* * *), 5 \%(* *)$ et $10 \%(*)$. Les régressions comprennent également la région de résidence des parents ( 8 modalités) et le fait de vivre dans une commune rurale. Pour les décompositions de variance, les familles ne contribuant que pour une observation à la vraisemblance du modèle ordonné sont exclues de l'échantillon.

Source : enquête Actifs financiers 1992, enquêtes Patrimoine 1998 et 2004.

La taille de la fratrie diminue fortement le niveau éducatif des enfants, conformément à l'arbitrage beckérien de type qualité-quantité. Les parents ne seront 
pas en mesure d'investir autant dans le capital humain de tous leurs enfants si ces derniers sont nombreux. À taille de fratrie donnée, il existe un avantage pour un enfant à avoir des sœurs plutôt que des frères. Plusieurs explications sont susceptibles de rendre compte de cet effet de composition par sexe (BarnetVerzat et Wolff [2003]). Tout d'abord, les parents peuvent favoriser les enfants pour lesquels la rentabilité du capital scolaire est la plus grande ${ }^{20}$. Ensuite, il peut exister des interactions entre l'éducation des collatéraux par l'intermédiaire de la fonction de production de capital humain. Si les enfants les plus âgés aident les plus jeunes à faire leurs devoirs, alors l'investissement accru des filles dans leurs études donne un avantage à avoir des sœurs. Enfin, les parents peuvent avoir des préférences différenciées pour les garçons et les filles.

Nous avons également pris en compte le rang de naissance comme facteur explicatif du diplôme. Une difficulté vient alors du fait que cette variable est corrélée avec la taille de la fratrie. Les enfants qui ont des rangs de naissance élevés appartiennent nécessairement à des fratries de grande taille. Afin de corriger cet effet de taille, nous utilisons le rang de naissance normalisé défini par Booth et Kee [2009], qui correspond au ratio du rang absolu $\mathrm{R}_{a b s}$ sur le rang absolu moyen. Si $N$ est la taille de la fratrie, alors le rang normalisé $\mathrm{R}_{n}$ est égal à $\mathrm{R}_{n}=\mathrm{R}_{a b s} /\left(\frac{\mathrm{N}+1}{2}\right)$. Par construction, ce rang normalisé ne dépend pas $\mathrm{du}$ nombre de frères et sœurs. D'après les résultats du tableau 3 , il existe une corrélation négative et significative entre le rang normalisé et le diplôme. Les aînés ont donc une trajectoire scolaire meilleure par rapport aux cadets en France.

En ce qui concerne les caractéristiques des parents, le niveau de diplôme des enfants est positivement corrélé avec l'âge du parent à la naissance de l'enfant (au seuil de $1 \%$ ). D'après les enquêtes Actifs financiers et Patrimoine, il est préjudiciable pour la réussite scolaire des enfants d'appartenir à une famille monoparentale ou recomposée, cet effet négatif étant beaucoup plus prononcé dans ce second cas de figure. Les changements au quotidien induits par une séparation, un veuvage ou une remise en couple viennent sans aucun doute altérer le suivi parental de l'effort scolaire fourni par les enfants. Il faut aussi tenir compte de la dégradation de la situation financière des familles dans de telles situations.

C'est surtout le niveau de capital humain des parents qui importe pour comprendre les trajectoires scolaires des enfants. Le diplôme obtenu par les enfants est d'autant plus important que le parent de référence est éduqué, notamment lorsqu'il a le bac et plus encore un diplôme supérieur au bac. Le fait d'appartenir à une famille riche est également corrélé positivement avec la trajectoire scolaire des enfants. Dans le modèle de Becker et Tomes [1986], lorsque les familles sont contraintes par la liquidité, l'investissement dans le capital humain est sous-optimal. Les parents sont en mesure d'investir dans l'éducation de leurs enfants jusqu'au niveau optimal seulement lorsqu'ils sont suffisamment riches.

Nous estimons ensuite un modèle avec pour variable dépendante le niveau d'éducation ordonné suivant cinq catégories : sans diplôme, inférieur au bac, bac, bac +2 , supérieur à bac +2 . Nous tenons compte du cas particulier des

20. Il est, dans ce cas, préférable d'avoir des sœurs plutôt que des frères dans la mesure où, à qualification égale, ce sont généralement les garçons qui ont les meilleures opportunités de rémunération sur le marché du travail. 
enfants interrogés dans l'enquête 1992 qui n'habitent plus chez leurs parents et ont un diplôme supérieur au bac. Les résultats sont présentés dans la colonne 2 du tableau 3. Globalement, ceci ne vient modifier qu'à la marge nos estimations. Le diplôme est en moyenne plus élevé pour les filles, pour les enfants appartenant aux cohortes de naissance les plus récentes, ayant peu de frères et sœurs et faisant partie des aînés de la fratrie. Le niveau d'éducation est également positivement corrélé avec l'âge du parent à la naissance de l'enfant, l'appartenance à une famille nucléaire, le diplôme du parent et le fait d'avoir des parents riches.

Enfin, nous ajoutons dans le modèle Probit ordonné à effets aléatoires une série de termes d'interactions croisant le sexe de l'enfant avec les différents facteurs explicatifs afin de mettre en évidence de possibles écarts entre les filles et les garçons. Les estimations présentées dans le tableau 3 apportent trois résultats complémentaires. Tout d'abord, les caractéristiques des parents n'ont pas d'incidence différenciée sur la trajectoire scolaire des garçons et des filles (à l'exception des diplômes CAP-BEP-BEPC au seuil de 5 \%). Ensuite, les filles appartenant aux cohortes les plus récentes ont clairement des niveaux d'éducation supérieurs aux garçons comme l'indiquent les effets croisés entre les cohortes de naissance et le sexe. L'écart entre garçons et filles devient significatif pour les enfants nés à partir de 1950. Enfin, le fait d'appartenir à une fratrie de grande taille s'avère plus préjudiciable pour les filles que pour les garçons ${ }^{21}$.

L'estimation de ces modèles ordonnés nous permet alors de procéder à l'analyse de variance, sur la base des niveaux éducatifs latents déduits des régressions précédentes. Dans la mesure où les inégalités de résultats entre enfants uniques correspondent nécessairement à des inégalités inter-familles, nous avons au préalable réestimé les régressions du tableau 3 en excluant les familles ne contribuant que pour une observation à l'échantillon. Cette sélection, qui revient à éliminer 3693 enfants de l'échantillon, ne modifie aucun de nos résultats précédents.

Nous trouvons que l'hétérogénéité d'éducation observée en France est davantage liée à des écarts entre les familles qu'à des écarts entre frères et sœurs au sein d'une même famille. Les variances inter et intra représentent respectivement $66,5 \%$ et $33,5 \%$ de la variance totale dans la spécification avec quatre niveaux d'éducation. Pour les écarts entre familles, les déterminants pris en compte dans les régressions expliquent $53,5 \%$ de la variance totale inter $(35,6 \% / 66,5 \%$ $=53,5 \%$ ). À l'inverse, il est très difficile d'expliquer les inégalités entre frères et sœurs, la partie expliquée de la variance intra restant très faible $(0,4 \% / 33,5 \%$ $=1,2 \%$ ). Des résultats très similaires sont obtenus pour la décomposition de variance avec cinq niveaux d'éducation (en tenant compte du cas particulier des enfants hors domicile diplômés du supérieur en 1992) ou bien encore lorsque les effets croisés sont introduits dans la liste des régresseurs.

Le poids relatif des composantes expliquée et inexpliquée de la variance intra n'est guère surprenant. Une limite des enquêtes Actifs financiers et Patrimoine tient à ce qu'elles ne permettent pas de prendre en compte les capacités intrinsèques de chaque enfant (leur talent) et leur effort. Ces variables n'étant pas observées dans nos données, elles sont intégrées à travers le terme d'erreur $\xi_{j i}$ dans notre spécification. À défaut d'avoir une mesure des aptitudes et des

21. Cet effet différencié de la taille de la fratrie suivant le genre apparaît difficile à expliquer. Il se peut qu'une préférence parentale plus grande pour les garçons soit à l'œuvre dans cette configuration. 
efforts individuels, il est particulièrement difficile de rendre compte des facteurs expliquant les écarts scolaires entre les frères et sœurs. Si cette limite affecte le poids relatif des deux composantes de la variance intra, elle n'a toutefois pas d'incidence sur les contributions respectives des variances inter et intra.

\section{Analyses de robustesse}

\section{Familles nucléaires versus familles monoparentales/recomposées}

Compte tenu de l'incidence des ruptures et recompositions familiales sur le diplôme des enfants, il est intéressant de savoir si l'hétérogénéité d'éducation diffère entre les familles nucléaires et les autres configurations. Pour ces dernières, l'existence de chocs familiaux (rupture, veuvage) peut venir aggraver la situation des plus jeunes enfants qui seront sans doute les plus vulnérables. Dans le même temps, si la situation financière du parent en charge des enfants se dégrade fortement, cela peut venir mettre un terme à la poursuite d'études des enfants plus âgés pour lesquels les dépenses privées d'éducation vont être plus importantes. Dans le tableau 4, nous présentons les estimations obtenues respectivement pour les familles nucléaires et les familles monoparentales et recomposées.

Tableau 4. Modèles Probit ordonnés à effets aléatoires expliquant l'éducation des enfants, par type de famille

\begin{tabular}{|c|c|c|c|c|c|}
\hline \multicolumn{2}{|c|}{ Variables } & $\begin{array}{c}\text { Familles } \\
\text { nucléaires }\end{array}$ & $\begin{array}{c}\text { Familles } \\
\text { monoparentales } \\
\text { et recomposées }\end{array}$ & $\begin{array}{c}\text { Familles } \\
\text { pauvres }\end{array}$ & $\begin{array}{l}\text { Familles } \\
\text { riches }\end{array}$ \\
\hline \multicolumn{6}{|c|}{ Caractéristiques des enfants } \\
\hline \multicolumn{2}{|l|}{ Sexe féminin } & $\begin{array}{c}0,225 * * * \\
(9,85)\end{array}$ & $\begin{array}{c}0,138 * * * \\
(4,57)\end{array}$ & $\begin{array}{c}0,176^{* * * *} \\
(6,96)\end{array}$ & $\begin{array}{c}0,207 * * * \\
(7,75)\end{array}$ \\
\hline \multirow[t]{7}{*}{$\begin{array}{l}\text { Cohorte } \\
\text { (réf. : < 1945) }\end{array}$} & $1945-1949$ & $\begin{array}{c}0,162 * \\
(1,95)\end{array}$ & $\begin{array}{c}0,126^{* *} \\
(2,13)\end{array}$ & $\begin{array}{l}0,050 \\
(0,80)\end{array}$ & $\begin{array}{c}0,261 * * * \\
(3,68)\end{array}$ \\
\hline & $1950-1954$ & $\begin{array}{c}0,223 * * * \\
(2,76)\end{array}$ & $\begin{array}{c}0,237 * * * \\
(3,94)\end{array}$ & $\begin{array}{c}0,154 * * \\
(2,47)\end{array}$ & $\begin{array}{c}0,329 * * * \\
(4,68)\end{array}$ \\
\hline & $1955-1959$ & $\begin{array}{c}0,253 * * * \\
(3,14)\end{array}$ & $\begin{array}{c}0,240 * * * \\
(3,79)\end{array}$ & $\begin{array}{c}0,137 * * \\
(2,16)\end{array}$ & $\begin{array}{c}0,406 * * * \\
(5,71)\end{array}$ \\
\hline & $1960-1964$ & $\begin{array}{c}0,210 * * * \\
(2,60)\end{array}$ & $\begin{array}{c}0,176^{* * * *} \\
(2,64)\end{array}$ & $\begin{array}{l}0,103 \\
(1,58)\end{array}$ & $\begin{array}{c}0,363 * * * \\
(5,01)\end{array}$ \\
\hline & $1965-1969$ & $\begin{array}{c}0,362 * * * \\
(4,40)\end{array}$ & $\begin{array}{c}0,275 * * * \\
(3,90)\end{array}$ & $\begin{array}{c}0,192 * * * \\
(2,85)\end{array}$ & $\begin{array}{c}0,583 * * * \\
(7,81)\end{array}$ \\
\hline & 1970-1974 & $\begin{array}{c}0,616^{* * * *} \\
(7,25)\end{array}$ & $\begin{array}{c}0,419 * * * \\
(5,45)\end{array}$ & $\begin{array}{c}0,442 * * * \\
\quad(6,10)\end{array}$ & $\begin{array}{c}0,793 * * * \\
(10,15)\end{array}$ \\
\hline & $1975+$ & $\begin{array}{c}0,791 * * * \\
(8,26)\end{array}$ & $\begin{array}{c}0,508 * * * \\
(5,20)\end{array}$ & $\begin{array}{c}0,565 * * * \\
(6,01)\end{array}$ & $\begin{array}{c}0,947 * * * \\
(10,59)\end{array}$ \\
\hline \multicolumn{2}{|c|}{ Nombre de frères et sœurs } & $\begin{array}{c}-0,153 * * * \\
(-10,98)\end{array}$ & $\begin{array}{c}-0,197 * * * \\
(-11,27)\end{array}$ & $\begin{array}{c}-0,195 * * * \\
(-13,43)\end{array}$ & $\begin{array}{c}-0,169 * * * \\
(-10,30)\end{array}$ \\
\hline \multicolumn{2}{|c|}{ Nombre de sœurs } & $\begin{array}{c}0,038 * * \\
(2,05)\end{array}$ & $\begin{array}{c}0,070 * * * \\
(2,86)\end{array}$ & $\begin{array}{c}0,056^{* * *} * \\
(2,73)\end{array}$ & $\begin{array}{c}0,043^{*} \\
(1,91)\end{array}$ \\
\hline
\end{tabular}




\begin{tabular}{|c|c|c|c|c|c|}
\hline \multicolumn{2}{|c|}{ Variables } & $\begin{array}{l}\text { Familles } \\
\text { nucléaires }\end{array}$ & $\begin{array}{c}\text { Familles } \\
\text { monoparentales } \\
\text { et recomposées }\end{array}$ & $\begin{array}{l}\text { Familles } \\
\text { pauvres }\end{array}$ & $\begin{array}{l}\text { Familles } \\
\text { riches }\end{array}$ \\
\hline \multicolumn{2}{|l|}{ Rang normalisé } & $\begin{array}{c}-0,356 * * * \\
(-10,05)\end{array}$ & $\begin{array}{c}-0,337 * * * \\
(-7,52)\end{array}$ & $\begin{array}{c}-0,351 * * * \\
(-9,52)\end{array}$ & $\begin{array}{c}-0,334 * * * \\
(-8,45)\end{array}$ \\
\hline \multicolumn{6}{|c|}{ Caractéristiques de la personne de référence } \\
\hline \multicolumn{2}{|c|}{$\begin{array}{l}\text { Âge du père/du parent à la naissance } \\
\text { de l'enfant }\end{array}$} & $\begin{array}{l}0,022 * * * \\
(5,26)\end{array}$ & $\begin{array}{c}0,033 * * * \\
(8,11)\end{array}$ & $\begin{array}{c}0,033 * * * \\
(9,83)\end{array}$ & $\begin{array}{l}0,026^{* * *} \\
(7,43)\end{array}$ \\
\hline \multicolumn{2}{|c|}{$\begin{array}{l}\text { Âge de la mère à la naissance } \\
\text { de l'enfant }\end{array}$} & $\begin{array}{c}0,011 * * \\
(2,36)\end{array}$ & & & \\
\hline \multicolumn{2}{|c|}{ Famille monoparentale } & & & $\begin{array}{c}-0,121 * * * \\
(-3,21) \\
\end{array}$ & $\begin{array}{c}-0,124 * * * \\
(-3,21)\end{array}$ \\
\hline \multicolumn{2}{|c|}{ Famille recomposée } & & $\begin{array}{c}-0,328 * * * \\
(-5,12)\end{array}$ & $\begin{array}{c}-0,458 * * * \\
(-5,74)\end{array}$ & $\begin{array}{c}-0,439 * * * \\
(-5,52)\end{array}$ \\
\hline \multicolumn{2}{|c|}{$\begin{array}{l}\text { Education du père/du parent CEP } \\
\text { (réf. : sans) }\end{array}$} & $\begin{array}{c}0,784 * * * \\
(18,65)\end{array}$ & $\begin{array}{c}0,629 * * * \\
(12,07)\end{array}$ & $\begin{array}{c}0,656^{* * *} \\
(15,49)\end{array}$ & $\begin{array}{c}0,514 * * * \\
(10,23)\end{array}$ \\
\hline \multicolumn{2}{|r|}{ CAP-BEP-BEPC } & $\begin{array}{c}1,318 * * * \\
(26,55)\end{array}$ & $\begin{array}{l}1,011 * * * \\
(16,31)\end{array}$ & $\begin{array}{l}0,982 * * * \\
(19,78)\end{array}$ & $\begin{array}{l}0,909 * * * \\
(16,91)\end{array}$ \\
\hline \multicolumn{2}{|r|}{$\mathrm{Bac}$} & $\begin{array}{c}2,047 * * * \\
(30,68)\end{array}$ & $\begin{array}{c}1,757 * * * \\
(21,66)\end{array}$ & $\begin{array}{c}1,706 * * * \\
(23,47)\end{array}$ & $\begin{array}{c}1,577 * * * \\
(23,45)\end{array}$ \\
\hline \multicolumn{2}{|r|}{$>$ bac } & $\begin{array}{c}2,732 * * * \\
(41,96)\end{array}$ & $\begin{array}{c}2,413 * * * \\
(27,55)\end{array}$ & $\begin{array}{c}2,389 * * * \\
(28,80)\end{array}$ & $\begin{array}{c}2,190 * * * \\
(34,78)\end{array}$ \\
\hline \multicolumn{2}{|c|}{$\begin{array}{l}\text { Différentiel d'éducation Père }=\text { mère } \\
\text { (Père }>\text { mère) }\end{array}$} & $\begin{array}{l}0,447 * * * \\
(12,18)\end{array}$ & & & \\
\hline \multicolumn{2}{|r|}{ Père $<$ mère } & $\begin{array}{c}0,907 * * * \\
(18,55)\end{array}$ & & & \\
\hline \multicolumn{2}{|c|}{ Famille riche (transferts aux enfants) } & $\begin{array}{c}0,384 * * * \\
(12,77)\end{array}$ & $\begin{array}{c}0,357 * * * \\
(8,43)\end{array}$ & & \\
\hline \multirow[t]{3}{*}{ Variance inter } & Expliquée & $\begin{array}{c}1,069 \\
(37,7 \%)\end{array}$ & $0,925(31,0 \%)$ & $\begin{array}{c}0,736 \\
(27,4 \%)\end{array}$ & $\begin{array}{c}0,927 \\
(33,2 \%)\end{array}$ \\
\hline & Inexpliquée & $\begin{array}{c}0,750 \\
(26,5 \%)\end{array}$ & $1,053(35,2 \%)$ & $\begin{array}{c}0,936 \\
(34,9 \%)\end{array}$ & $\begin{array}{c}0,851 \\
(30,5 \%)\end{array}$ \\
\hline & Total & $\begin{array}{c}1,819 \\
(64,2 \%)\end{array}$ & $1,978(66,2 \%)$ & $\begin{array}{c}1,672 \\
(62,3 \%)\end{array}$ & $\begin{array}{c}1,778 \\
(63,7 \%)\end{array}$ \\
\hline \multirow[t]{3}{*}{ Variance intra } & Expliquée & $\begin{array}{c}0,013 \\
(0,5 \%)\end{array}$ & $0,009(0,3 \%)$ & $\begin{array}{c}0,010 \\
(0,4 \%)\end{array}$ & $\begin{array}{c}0,014 \\
(0,5 \%)\end{array}$ \\
\hline & Inexpliquée & $\begin{array}{c}1,000 \\
(35,3 \%)\end{array}$ & $1,000(33,5 \%)$ & $\begin{array}{c}1,000 \\
(37,3 \%)\end{array}$ & $\begin{array}{c}1,000 \\
(35,8 \%)\end{array}$ \\
\hline & Total & $\begin{array}{c}1,013 \\
(35,8 \%)\end{array}$ & $1,009(33,8 \%)$ & $\begin{array}{c}1,010 \\
(37,7 \%)\end{array}$ & $\begin{array}{c}1,014 \\
(36,3 \%)\end{array}$ \\
\hline \multicolumn{2}{|c|}{ Nombre d'observations } & 16547 & 10637 & 14439 & 12758 \\
\hline \multicolumn{2}{|c|}{ Nombre de familles } & 7341 & 4447 & 5930 & 5764 \\
\hline \multicolumn{2}{|c|}{ Log vraisemblance } & $-18959,5$ & $-12077,9$ & $-16216,5$ & $-15030,0$ \\
\hline \multicolumn{6}{|c|}{$\begin{array}{l}\text { Les régressions estimées sont des modèles Probit ordonnés à effets aléatoires. Les seuils de significativité retenus } \\
\text { sont respectivement de } 1 \%(* * *), 5 \%(* *) \text { et } 10 \%(*) \text {. Les régressions comprennent également la région de } \\
\text { résidence des parents }(8 \text { modalités) et le fait de vivre dans une commune rurale. Pour les décompositions de } \\
\text { variance, les familles ne contribuant que pour une observation à la vraisemblance du modèle ordonné sont } \\
\text { exclues de l'échantillon. } \\
\text { Source : enquête Actifs financiers } 1992 \text {, enquêtes Patrimoine } 1998 \text { et } 2004 \text {. }\end{array}$} \\
\hline
\end{tabular}


Pour les familles nucléaires, nous prenons en compte à la fois les caractéristiques du père et de la mère dans l'explication du parcours éducatif des enfants ${ }^{22}$. Le diplôme est positivement corrélé à la fois avec l'âge du père et de la mère, les coefficients associés n'étant pas significativement différents. Le diplôme de l'enfant augmente fortement avec celui du père, surtout lorsque ce dernier a le bac ou un diplôme supérieur. À diplôme du père donné, la position relative de la mère en termes d'éducation exerce également une incidence significative sur le parcours scolaire des enfants. Ceux-ci sont en moyenne plus éduqués lorsque la mère a un diplôme supérieur à celui de son mari, et il en est de même (dans une moindre mesure) lorsque le père et la mère ont le même diplôme. Cet effet peut rendre compte de l'importance accrue de la transmission du capital humain pour les mères (Thomas [1994]) ou bien encore du temps plus important consacré par les mères que par les pères à s'occuper des devoirs de leurs enfants.

Si les variables explicatives retenues pour les parents et les enfants ont des effets très similaires sur l'éducation de ces derniers pour les deux types de familles, nous observons quelques différences intéressantes pour les décompositions de variance. D'un côté, le poids de la variance intra est de 2 points de pourcentage plus élevé pour les familles nucléaires (35,8\% au lieu de 33,8\%), ce qui correspond à une hétérogénéité plus grande dans les parcours des frères et sœurs. De l'autre, le poids de la variance inter inexpliquée est beaucoup plus élevé (près de 9 points) pour les familles monoparentales et recomposées. Ce résultat n'est guère surprenant puisque les trajectoires scolaires des enfants ont nécessairement été influencées par l'histoire familiale du parent de référence qui n'est pas prise en compte dans la régression ${ }^{23}$.

\section{Familles pauvres versus familles riches}

Nous avons également procédé à des estimations séparées pour les familles pauvres et les familles riches. Il semble difficile de savoir a priori quel peut être l'impact de la richesse parentale sur l'hétérogénéité d'éducation entre enfants. Pour les parents les plus fortunés, ceux qui ont des biens professionnels à transmettre à leurs enfants peuvent choisir d'investir davantage dans le capital humain de certains d'entre eux. Dans le même temps, ces parents ont aussi davantage de moyens pour assurer le financement d'études longues à tous leurs enfants. Dans les familles pauvres, le poids des contraintes de liquidité devrait être d'autant plus important que les parents ont de nombreux enfants scolarisés, ce qui peut favoriser les aînés et les derniers nés. Il est toutefois possible que les enfants ne bénéficient pas de conditions suffisamment favorables (à travers le capital humain et culturel de leurs parents) pour parvenir à des diplômes élevés ${ }^{24}$.

Les résultats indiqués dans le tableau 4 sont très semblables pour les deux types de familles. Les corrélations entre les caractéristiques des parents et des enfants et le diplôme de ces derniers sont très similaires pour les familles riches et pauvres, à l'exception des effets de cohortes. Ceux-ci apparaissent beaucoup

22. Nous sommes amenés à supprimer treize observations de l'échantillon pour lesquelles nous ne connaissons pas à la fois pour le père et la mère leur âge à la date de naissance des enfants et leur niveau d'éducation.

23. Ceci concerne, par exemple, la date de la séparation ou du veuvage s'il y a lieu (et l'âge des enfants à cette date) ou bien encore la situation socioéconomique de l'ancien conjoint ainsi que celle du nouveau conjoint lorsqu'il y a lieu.

24. Tous les enfants dans la fratrie seraient alors caractérisés par de faibles niveaux d'éducation. 
plus prononcés pour les familles riches. En ce qui concerne la décomposition de variance, le poids de la variance intra est un peu plus élevé pour les familles pauvres $(+1,4$ point de probabilité). L'hétérogénéité d'éducation entre frères et sœurs est donc un peu plus faible pour les familles les plus aisées. Par ailleurs, nous remarquons que la contribution des caractéristiques familiales (appréciée par la variance expliquée) est beaucoup plus importante lorsqu'il s'agit de comprendre les écarts de diplôme entre les enfants de familles différentes.

\section{Effets aléatoires versus effets fixes}

Notre mesure de l'hétérogénéité d'éducation des enfants repose sur l'estimation de modèles à effets aléatoires. Dans cette spécification, les effets familiaux $\delta_{j}$ sont supposés indépendants des variables explicatives $\mathrm{X}_{j i}$. Or, la pertinence de cette hypothèse apparaît discutable. Pour ne prendre qu'un seul exemple, les variables de cohortes de naissance ont de fortes chances d'être corrélées avec le capital culturel des parents, qui n'est pas observé dans les enquêtes ${ }^{25}$. Cette corrélation peut dès lors conduire à des estimations biaisées.

Dans de telles circonstances où $\mathrm{E}\left(\delta_{j} \mid \mathrm{X}_{j i}\right) \neq 0$, la solution proposée initialement par Mundlak [1978] pour le modèle linéaire et transposable au modèle Probit consiste à estimer un modèle à effets aléatoires corrélés. La régression comprend alors des variables explicatives supplémentaires qui correspondent aux moyennes par famille des variables $X_{j i}$ (Wooldridge [2002], p. 487-488). Soit $\overline{\mathrm{X}}_{j}=\frac{1}{\mathrm{~N}_{j}} \sum_{j} \mathrm{X}_{j i}$. Nous pouvons alors introduire ces moyennes dans la régression seulement pour les caractéristiques qui varient entre les différents enfants $i$ d'une famille $j$ donnée. Ceci ne concerne donc que les caractéristiques propres aux enfants et exclut toutes les variables parentales ainsi que la taille et la composition par sexe de la fratrie.

Afin d'apprécier les conséquences d'une possible corrélation entre $\mathrm{X}_{j i}$ et $\delta_{j}$, nous avons réestimé le modèle ordonné en incluant les moyennes calculées au niveau de la famille pour le sexe, la cohorte de naissance et le rang dans la fratrie pour les enfants. Soit $\gamma_{j}$ le vecteur de coefficients associés à ces moyennes $\overline{\mathrm{X}}_{j}$. Les résultats, non reportés, indiquent que l'hypothèse nulle $\mathrm{H}_{0}: \gamma_{j}=0$ est rejetée au seuil de $1 \%$ pour nos données ${ }^{26}$. La réussite scolaire est alors positivement corrélée avec les moyennes familiales pour le sexe féminin et les cohortes de naissances, tandis que la moyenne associée au rang de naissance n'est pas significative. L'ajout de ces moyennes ne modifie pas pour autant nos mesures de l'hétérogénéité d'éducation. Les poids des variances inter et intra sont de $65,8 \%$ et $34,2 \%$, soit un écart de 0,1 point avec le modèle à effets aléatoires comparables, décrit dans le tableau 3.

Pour finir, nous avons cherché à estimer un modèle ordonné à effets fixes pour regarder l'effet des caractéristiques des enfants sur la réussite scolaire au

25. Si l'on admet que le capital humain et le capital culturel des parents sont corrélés, alors la corrélation entre l'effet familial et l'appartenance à une cohorte de naissance récente devrait être positive : les enfants les plus jeunes ont en moyenne des parents plus diplômés compte tenu de l'expansion constante de l'enseignement.

26. Nous trouvons une valeur égale à 22,99 pour la statistique du $\mathrm{Chi}^{2}$ associée (avec neuf degrés de liberté). 
sein des fratries. Celles pour lesquelles tous les enfants ont le même niveau d'éducation ne sont désormais plus prises en compte. Nous suivons la procédure conditionnelle proposée par Das et Van Soest [1999]. Dans un premier temps, nous construisons des variables muettes $\mathrm{S}_{j i}^{k}$ telles que $\mathrm{S}_{j i}^{k}=1$ si $\mathrm{E}_{j i}>k$ et $\mathrm{S}_{j i}^{k}=0$ sinon, avec $k=1, \ldots, 4^{27}$. Nous estimons des modèles Logit conditionnels à la Chamberlain [1980] pour obtenir un estimateur à effets fixes $\beta^{k}$ de $\beta$. Dans un second temps, nous appliquons un estimateur à distance minimale pour former un estimateur restreint $\beta$ à partir de $\beta^{1}, \beta^{2}, \beta^{3}$ et $\beta^{4}$.

Les résultats du modèle à effets fixes sont présentés dans le tableau 5. Les caractéristiques parentales sont, par définition, exclues de ces régressions puisqu'elles sont communes aux frères et sœurs. Nous retrouvons alors les résultats mis en évidence dans le tableau 3 à partir d'une modélisation à effets aléatoires. Ce sont les filles, les enfants appartenant aux cohortes de naissance les plus récentes et les aînés qui réussissent le mieux en comparaison avec leurs frères et sœurs, tous ces effets étant significatifs au seuil de $1 \%$. Ces résultats s'observent aussi bien pour les familles riches que pour les familles pauvres.

Tableau 5. Modèles ordonnés à effets fixes expliquant l'éducation des enfants

\begin{tabular}{|c|c|c|c|c|}
\hline \multicolumn{2}{|c|}{ Variables } & $\begin{array}{c}\text { Ensemble } \\
\text { des familles }\end{array}$ & $\begin{array}{c}\text { Familles } \\
\text { pauvres }\end{array}$ & $\begin{array}{l}\text { Familles } \\
\text { riches }\end{array}$ \\
\hline \multicolumn{5}{|c|}{ Caractéristiques des enfants } \\
\hline Sexe féminin & & $\begin{array}{c}0,248 * * * \\
(9,32)\end{array}$ & $\begin{array}{c}0,232 * * * \\
(6,16)\end{array}$ & $\begin{array}{c}0,265 * * * \\
(7,01)\end{array}$ \\
\hline \multirow[t]{7}{*}{$\begin{array}{l}\text { Cohorte } \\
\text { (réf. : < 1945) }\end{array}$} & 1945-1949 & $\begin{array}{c}0,407 * * * \\
(4,47)\end{array}$ & $\begin{array}{c}0,297 * * \\
(2,41)\end{array}$ & $\begin{array}{c}0,556^{* * * *} \\
(4,07)\end{array}$ \\
\hline & $1950-1954$ & $\begin{array}{c}0,687 * * * \\
(6,61)\end{array}$ & $\begin{array}{c}0,735 * * * \\
(5,14)\end{array}$ & $\begin{array}{c}0,651 * * * \\
(4,28)\end{array}$ \\
\hline & 1955-1959 & $\begin{array}{c}0,938 * * * \\
(8,02)\end{array}$ & $\begin{array}{c}0,901 * * * \\
(5,59)\end{array}$ & $\begin{array}{c}1,001 * * * \\
(5,86)\end{array}$ \\
\hline & 1960-1964 & $\begin{array}{c}1,062 * * * \\
(8,00)\end{array}$ & $\begin{array}{c}1,105 * * * \\
(6,04)\end{array}$ & $\begin{array}{c}1,043 * * * \\
(5,36)\end{array}$ \\
\hline & 1965-1969 & $\begin{array}{c}1,517 * * * \\
(10,01)\end{array}$ & $\begin{array}{c}1,478 * * * \\
(7,06)\end{array}$ & $\begin{array}{c}1,577 * * * \\
(7,13)\end{array}$ \\
\hline & $1970-1974$ & $\begin{array}{c}2,006 * * * \\
(11,47)\end{array}$ & $\begin{array}{c}2,023 * * * \\
(8,35)\end{array}$ & $\begin{array}{c}2,012 * * * \\
(7,92)\end{array}$ \\
\hline & $1975+$ & $\begin{array}{c}2,227 * * * \\
(10,43)\end{array}$ & $\begin{array}{c}2,212 * * * \\
(7,24)\end{array}$ & $\begin{array}{c}2,242 * * * \\
(7,38)\end{array}$ \\
\hline \multicolumn{2}{|l|}{ Rang normalisé } & $\begin{array}{c}-0,436 * * * \\
(-9,23)\end{array}$ & $\begin{array}{c}-0,463 * * * \\
(-7,02)\end{array}$ & $\begin{array}{c}-0,407 * * * \\
(-6,00)\end{array}$ \\
\hline
\end{tabular}

Les régressions estimées sont des modèles ordonnés à effets fixes. Les seuils de significativité retenus sont respectivement de $1 \%(* *), 5 \%(* *)$ et $10 \%(*)$.

Source : enquête Actifs financiers 1992, enquêtes Patrimoine 1998 et 2004.

27. Compte tenu de l'impossibilité de savoir si $\mathrm{E}_{j i}=4$ ou $\mathrm{E}_{j i}=5$ pour les enfants hors domicile interrogés en 1992 , nous supposons que $\mathrm{S}_{j i}^{4}=1$ et $\mathrm{S}_{j i}^{5}=1$ pour ces enfants. Cette hypothèse est sans incidence sur nos résultats. Nous obtenons des résultats similaires lorsque nous estimons un modèle ordonné à effets fixes sur la base d'un diplôme ordonné suivant quatre catégories (les catégories $\mathrm{bac}+2$ et supérieur à bac +2 sont alors regroupées). Dans ce cas, l'estimateur à distance minimale s'appuie sur les estimations de trois modèles Logit conditionnels. 


\section{CONCLUSION}

Si les économistes ont largement documenté la transmission intergénérationnelle du capital humain, on sait, en revanche, assez peu de choses en France sur l'importance des inégalités intra-familiales d'éducation. Ceci s'explique sans aucun doute par la nécessité de disposer de données sur le diplôme obtenu par chacun des frères et sœurs.

Le recours aux enquêtes Actifs financiers et Patrimoine nous a permis de construire un échantillon appariant les caractéristiques des parents et celles de tous leurs enfants. Suivant Picard et Wolff [2010], la méthodologie mise en œuvre dans cet article nous permet de décomposer l'hétérogénéité d'éducation des enfants en différences de niveau éducatif moyen entre les familles, d'une part, et en différences de niveau éducatif entre les différents frères et sœurs au sein d'une famille, d'autre part. Les principaux résultats de l'analyse économétrique, qui sont cohérents avec les travaux réalisés à ce jour en ce qui concerne les déterminants de la réussite scolaire, sont les suivants.

Tout d'abord, près des deux tiers de l'hétérogénéité d'éducation en France correspondent à des différences entre les familles, affectant également tous les enfants de la famille considérée. En comparaison, les écarts entre les frères et sœurs sont presque deux fois moins importants. Ensuite, si les variables explicatives retenues rendent assez bien compte des inégalités moyennes d'éducation entre les familles, il est très difficile d'expliquer les inégalités qui peuvent exister au sein des fratries. Ceci est assurément lié au choix des variables explicatives retenues, puisque seuls le sexe, la cohorte de naissance et le rang peuvent varier entre enfants d'une même fratrie. Enfin, l'estimation d'un modèle ordonné à effets fixes montre que, parmi les frères et sœurs, ce sont les filles, les enfants parmi les cohortes les plus récentes et les aînés des fratries qui réussissent relativement mieux.

$\mathrm{Du}$ point de vue de la politique publique, il paraît important de mieux comprendre les facteurs qui influencent les écarts d'éducation entre frères et sœurs quand il y a lieu. Si les inégalités observées au sein des fratries résultent de choix délibérés des parents de privilégier tel ou tel enfant, alors des mesures correctrices peuvent être envisagées dans une perspective de réduction de cette hétérogénéité. Il est toutefois possible que les frères et sœurs ne parviennent pas tous au même diplôme, soit parce qu'ils ne fournissent pas le même effort au cours de leur cursus (effort qui n'est pas observé dans les enquêtes utilisées), soit pour de simples considérations chronologiques (Maurin et McNally [2008]). Les cadets peuvent rencontrer un système éducatif devenu moins sélectif par rapport à leurs aînés ou bénéficier d'un effort public accru dans la prise en charge des dépenses d'éducation. Il serait assurément pertinent de savoir si les parents ajustent leurs investissements dans le capital humain de leurs enfants dans de telles circonstances.

\section{RÉFÉRENCES BIBLIOGRAPHIQUES}

Albouy V. et Tavan C. [2008], « Accès à l'enseignement supérieur en France : une démocratisation réelle, mais de faible ampleur », Économie et statistique, 410, p. 3-22. 
BARNET-VERZAT C. et WOLFF F.-C. [2003], « Choix d'éducation et composition par sexe de la fratrie », Économie et prévision, 157, p. 97-118.

BAUdelot C. et Establet R. [1992], Allez les filles !, Paris, Le Seuil.

BECKER G.S. et TOMES N. [1986], " Human capital and the rise and fall of families », Journal of Labor Economics, 4, p. S1-S39.

Behrman J., Pollak R. et Taubman P. [1995], From Parent to Child. Intrahousehold Allocation and Intergenerational Relations in the United States, Chicago, University of Chicago Press.

BERHMAN J. [1997], « Intrahousehold distribution and the family », dans ROSENZWEIG M.R. et StARK O. (dir.), Handbook of Population and Family Economics, vol. 1A, Amsterdam, Boston, Paris, Elsevier, p. 125-187.

BJÖRKLUND A. et SALVANES K.G. [2011], « Education and family background: Mechanisms and policies », dans HANUSHEK E.A., MACHIN S. et WOESSMANN L. (dir.), Handbook of the Economics of Education, Amsterdam, Boston, Heidelberg, Elsevier North-Holland, p. 201-247.

BLack S.E., DeVEREUX P.J. et SALVANES K.G. [2005a], « Why the apple doesn't fall far: Understanding intergenerational transmission of human capital », American Economic Review, 95, p. 437-449.

Black S.E., Devereux P.J. et Salvanes K.G. [2005b], « The more the merrier? The effect of family composition on children's outcomes ", Quarterly Journal of Economics, 120, p. 669-700.

Bоотн A. et KeE H. [2009], « Birth order matters: The effect of family size and birth order on educational attainment », Journal of Population Economics, 22, p. 367-397.

BUTCHER K.F. et CASE A. [1994], « The effect of sibling sex composition on women's education and earnings », Quarterly Journal of Economics, 109, p. 531-563.

BUTLER L.S. et MOFFITT R. [1982], « A computationally efficient quadrature procedure for the one factor multinomial Probit model », Econometrica, 50, p. 761-764.

CARD D. [2001], "Estimating the return to schooling: Progress on some persistent econometric problems », Econometrica, 69, p. 1127-1160.

ChAmBERLAIN G. [1980], " Analysis of covariance with qualitative data », Review of Economic Studies, 47, p. 225-238.

DAS M., VAN Soest A. [1999], " A panel data model for subjective information on household income growth », Journal of Economic Behavior and Organization, 40, p. $409-426$.

DUÉE M. [2005], «L'impact du chômage des parents sur le devenir scolaire des enfants », Revue économique, 56, p. 637-645.

Duru-Bellat M., KiefFer A. et MARry C. [2001], « La dynamique des scolarités des filles : le double handicap questionné », Revue française de sociologie, 42, p. 251-280.

EJRNAES M. et PÖRTNER C. [2004], « Birth order and the intra-household allocation of time and education », Review of Economics and Statistics, 86, p. 1008-1119.

ESTABLET R. [1988], « Subversion dans la reproduction scolaire », Revue économique, 39, p. 71-91.

FABre A. et Moullet S. [2004], « Externalités de l'éducation et mobilité intergénérationnelle : application au cas français », Économie et prévision, 166, p. 19-37.

Frechette G.R. [2001], « Random effects ordered Probit », Stata Technical Bulletin, 159, p. 23-27.

GARG A. et MoRDUCH J. [1998], « Sibling rivalry and the gender gap: Evidence from child health outcomes in Ghana », Journal of Population Economics, 11, p. 471-493.

GARY-Bobo R., PiCARD N. et Prieto A. [2006], « Birth order and sibship sex composition as instruments in the study of education and earnings ", CEPR Discussion Paper, 5514.

GouX D. et MAURIN E. [1995], « Origine sociale et destinée scolaire. L'inégalité des chances devant l'enseignement à travers les enquêtes FQP 1970, 1977, 1985 et 1993 », Revue française de sociologie, 36, p. 81-121. 
GOUX D. et MAURIN E. [1997], «Démocratisation de l'école et persistance des inégalités », Économie et statistique, 306, p. 27-39.

GOUX D. et MAURIN E. [2000], «La persistance du lien entre pauvreté et échec scolaire», in France portrait social, Insee, p. 87-98.

GouX D. et MAURIN E. [2005], " The effect of overcrowded housing on children's performance at school », Journal of Public Economics, 89, p. 797-819.

HANUSHEK E.A. et WOESSMANN L. [2011], «The economics of international differences in educational achievement ", dans HANUSHEK E.A., MACHIN S. et WOESSMANN L. (dir.), Handbook of the Economics of Education, Amsterdam, Boston, Heidelberg, Elsevier North Holland, p. 89-200.

HAUSER R.M. et KUO H.H.D. [1998], « Does the gender composition of sibships affect women's educational attainment? », Journal of Human Resources, 33, p. 644-657.

HAVEMAN R. et WOLFE B. [1995], « The determinants of childrens' attainments: A review of methods and findings ", Journal of Economic Literature, 33, p. 1829-1878.

KAESTNER R. [1997], « Are brothers really better? Sibling sex composition and educational achievement revisited », Journal of Human Resources, 32, p. 250-284.

MAGNAC T. et THESMAR D. [2002], «Évaluation d'une politique éducative : la montée de la scolarisation en France 1982-1993 », Annales d'économie et de statistique, 65, p. 1-33.

MAURIN E. [2002], « The impact of parental income on early schooling transitions: A re-examination using data over three generations », Journal of Public Economics, 85, p. 301-332.

MAURIN E. et MCNALLY S. [2008], « Vive la Révolution! Long term returns of 1968 to the angry students ", Journal of Labor Economics, 26, p. 1-35.

MUNDLAK Y. [1978], " On the pooling of time series and cross section data ", Econometrica, 46, p. 69-85.

PICARD N. et WOLFF F.-C. [2010], « Measuring educational inequalities: A method and an application to Albania », Journal of Population Economics, 23, p. 989-1023.

THOMAS D. [1994], « Like father, like son; Like mother, like daughter: Parental resources and child height », Journal of Human Resources, 29, p. 950-988.

WOLFF F.-C. [2012], « Le rang dans la fratrie influence-t-il le niveau d'éducation ? », Informations sociales, 173, p. 62-69.

Wooldridge J.M. [2002], Econometric Analysis of Cross Section and Panel Data, Cambridge (Mass.), The MIT Press. 
